\title{
Symmetry of Magnetostatic Fields Generated by Toroidal Helicoidal Magnets
}

\author{
Roberto Muscia \\ Department of Engineering and Architecture, University of Trieste, Trieste 34127, Italy
}

In this paper, the axial symmetry of the magnetic field generated by a permanent magnet of helicoidal toroidal kind is shown. In the first part of this paper, we illustrate the shape of the magnet and the number of areas where the field is calculated to demonstrate the symmetry. We define quantitatively the size of the toroidal helical magnet and the regions where the magnetostatic field is evaluated. The field is carried out for each angular sector that represents the regions where the magnetic flux density is computed. This calculation is performed with reference to a matrix of points belonging to each sector. Two sets of evaluations are performed. The first one is referred to a less dense matrix of points relative to all the regions. The aim of this computation is to demonstrate the axial symmetry of the field. The second set of calculations concerns the field evaluation using a much higher dense matrix of points. Using these data, we are able to interpolate the same field with a high precision. This second evaluation of the field is carried out with reference to only the flat region facing the first coil of the helical toroidal magnet. The use of an interpolation surface through the final points of the magnetic induction vectors previously computed allows a very fast evaluation of the field virtually in all the infinite points of the angular sector. The symmetry enables us to drastically reduce the time computation of the magnetostatic field in the points of interest.

Index Terms-Geometry, helix, magnetic charge, magnetostatic, permanent magnets, symmetry, torus.

\section{INTRODUCTION}

$\mathbf{T}$ HE study of new highly efficient electrical machines can require the development of unconventional design configurations. These configurations can prove their validity only if based on adequate evaluation studies of magnetic fields that are typical of the machines studied. In Fig. 1, an example of these configurations is illustrated by a conceptual model. The rotor element denoted by $\mathrm{A}$ is driven by a toroidal curvilinear screw B. In order to obtain the correct positioning of the part A in relation to the toroidal helicoidal permanent magnets $\mathrm{C}$, the same part A can rotate around the pin D. The pin D is joined to the part $\mathrm{E}$ where six bearings $\mathrm{F}$ are assembled. Between the magnets $\mathrm{C}$ and the surface of $\mathrm{A}$ opposite $\mathrm{C}$, there is an air gap. The various positions of this surface represent the areas where the magnetic field has to be computed. The coupling between the toroidal curvilinear screw B and the part E could also be obtained by magnetic repulsion. Fig. 2 shows a 3-D virtual model of the coupling solution by permanent magnets. This is another possible application of the magnetic field generated by toroidal helicoidal permanent magnets. The device can be manufactured by assembling six sets $1,2, \ldots, 6$ of very small parallelepiped neodymium magnets that approximate the toroidal helicoidal surfaces of the three threads of the curvilinear screw $\mathrm{B}$. Therefore, the six bearings $\mathrm{F}$ shown in Fig. 1 will be replaced by six sets $1^{\prime}, 2^{\prime}, \ldots, 6^{\prime}$ of neodymium magnets arranged with the same toroidal helicoidal configuration of B. Each of these sets defines two air gaps between the screw thread and the correspondent female thread. For these particular configurations, at the present time

Manuscript received January 30, 2015; revised April 22, 2015; accepted June 1, 2015. Date of publication June 10, 2015; date of current version September 16, 2015. Corresponding author: R. Muscia (e-mail: muscia@units.it).

Color versions of one or more of the figures in this paper are available online at http://ieeexplore.ieee.org.

Digital Object Identifier 10.1109/TMAG.2015.2443719

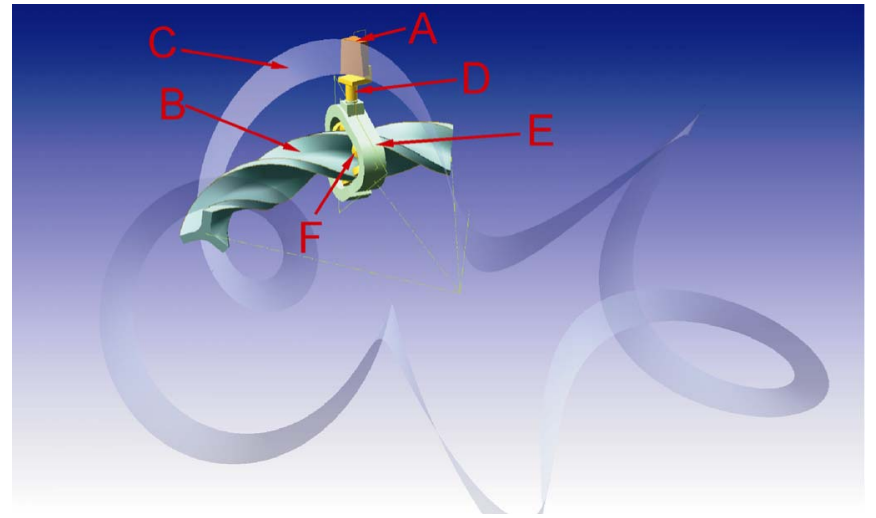

Fig. 1. Conceptual model of an unconventional design configuration of an electrical machine.
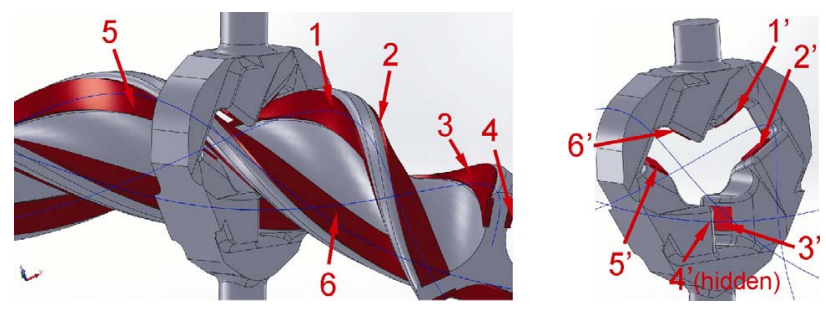

Fig. 2. Magnetic toroidal curvilinear screw.

in progress, the fields generated by permanent magnets and currents must be accurately simulated. As a matter of fact, the fitness of the design and the correct analysis of the device, functioning strongly depends on the precision with which the forces that the stator and rotor mutual exchange are calculated. This accuracy is a function of the precision with which we evaluate the magnetic field on the surfaces where these forces will be calculated. These observations are especially valid in relation to the use of the method of the magnetic 
charges [1]-[5] which allows the computation of the magnetic field, forces, and moments that affect the device study. The method of magnetic charges can be very accurate without the high computational time cost. Unfortunately, in general, this feature is not found when we perform simulations by the finiteelement method (FEM). Such accuracy can be very useful if we want to evaluate small increments of the electric machine efficiency that we are studying. Concerning this issue, it is well known that the use of FEM in electromagnetics (as in other subjects) introduces errors related to the discretization and numerical problems can also arise because of the large size of the algebraic systems that have to be solved. In relation to the reliability of the FEM simulations regarding this topic, various indices of fitness have been proposed [6]-[12], but the problem of the precision required does not make this method suitable for performing accurate evaluations of the magnetic field relative to unconventional configurations of electrical machines. Furthermore, the option to increase the FEM meshing has its limits [13], because an excessive discretization greatly increases the computation time and can cause a numerical ill-conditioning that produces errors greater than those associated with a less dense mesh. These kinds of problems also arise in other fields, for example, when magnetic fields in synchrotron light sources [14], tokamaks [15]-[19], stellarator [20]-[24], and hybrid tokamak-stellarator [25] have to be evaluated with high precision to study the controlled nuclear fusion. In the tokamaks, an axial toroidal magnetic field is created by vertical coils. The primary of a large transformer induces an axial toroidal current in the plasma acting as the secondary of the same transformer. This plasma current creates a poloidal magnetic field perpendicular to the axial toroidal magnetic field and the combination of these two fields generates a helical toroidal magnetic field which contains the extremely hot plasma by holding it away from the wall of the torus. From a magnetic point of view, also in the stellarator, the final result is a helical toroidal magnetic field that avoids contact of the plasma with the wall of the torus. Nevertheless, due to their inherently three-dimensional nature, stellarators rely heavily on the numerical simulation and the computation of the helical toroidal field. The optimized design and physics analysis of this device would not be possible without access to high-performance parallel computers and the magnetic field has to be evaluated with a precision far higher than that requested for the tokamaks. Therefore, the knowledge a priori of a particular axial symmetry of a helical toroidal magnetic field is certainly helpful, because it enables one to limit the zones where the computation has to be performed (the calculated magnetic field in the areas of interest is replicated with an axial symmetry in all the other regions of the device). This fact reduces the time computation and allows to increase the accuracy of the computation. Therefore, for example, with reference to applications as the development of new configuration of highly efficient electrical machines and easing of the optimal magnetic field computation in tokamaks and stellarator, to know a priori a particular axial symmetry of the helical magnetic toroidal field is surely advantageous. In relation to the previous observations, in order to demonstrate and characterize the axial symmetry of a helical

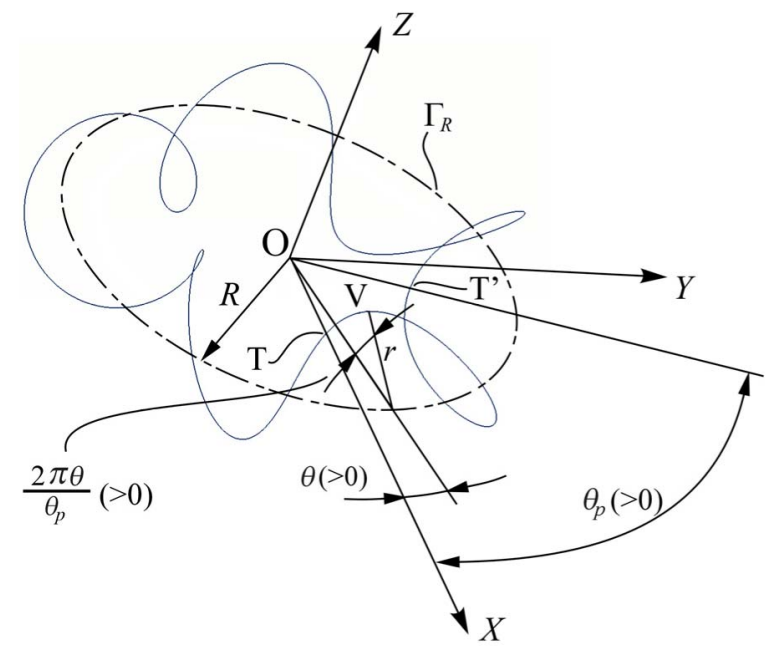

Fig. 3. Toroidal helix.

magnetic toroidal field, in this paper, we study the properties of the magnetic field produced by a particular configuration of a permanent magnet using the method of the magnetic charges. These properties will be evaluated by considering a simplifying hypothesis, i.e., the modulus of the magnetization vector $\mathbf{M}$ of the toroidal helicoidal permanent magnets is constant. With particular reference to the development of new configurations of electrical machines, this hypothesis represents only the first choice that can be advantageous in order to reduce the computational cost regardless of the method that we used to evaluate the magnetic field. When a reasonable configuration of the system has been found, we will apply more complex models to consider the possible influence of the nonlinear magnetization characteristic of the helical magnet and the consequent saturation effects. These effects have been studied in-depth in relation to electric machines of various kinds [26]-[28] and to the nonlinear demagnetization of permanent magnets [29], [30]. The problem of the demagnetization rises from the operating conditions and may have great influence on the performances of the machine, because the magnetic characteristic becomes strongly nonlinear. Usually, the saturation effects and demagnetization reduce the performances and the efficiency of the traditional electric devices. Likewise, also in the development of new electric machines, these effects have to be carefully evaluated as soon as a new promising design configuration is found using the simplifying hypothesis based on the constant modulus of magnetization M. With reference to these assumptions, in [31], a preliminary study of a helicoidal toroidal magnet of which the mean helix is shown in Fig. 3 has been developed. In the present case study, unlike the previous one, a particular axial symmetry of the magnetostatic field generated by the magnet is quantitatively proved by computing the same field on certain surfaces. These regions identify the current positions of a rotor element during the operation of the machine (see Fig. 1). The architecture development of the system is in progress, but the evaluation of the field in correspondence to such areas is the first step to understand if the device will work correctly. In relation to the calculation of this field, from a computational point of view, it is extremely advantageous to use a particular 
symmetry of the same field. This symmetry, as it will be illustrated quantitatively in the continuation of the present case study, allows us to limit the integration calculations for the evaluation of the field only in correspondence to the regions of interest relating to a single coil. The configuration of the magnet is such that the calculated magnetic field in the areas of interest is replicated with an axial symmetry in all the other homologous regions relative to the other coils. Therefore, using this symmetry, if the magnet is manufactured by $n$ helical toroidal coils, to get the field in all the $n$ regions of interest, the integration calculations can be performed only for the regions related to a single coil. In the remaining $n-1$ regions, the magnetic induction vector will be rotated around the axis of the toroidal helical geometry, exactly happens with the simple cylindrical magnets. In the following sections II-VI, we describe the mathematical formulation of the geometry of the magnet, the distribution of the relative magnetization vector $\mathbf{M}$, and the position of the regions where the field is calculated.

\section{Geometrical Configuration of the Magnet}

The procedure to obtain the shape of the helical toroidal magnet consists of the following steps.

1) The definition of a toroidal helix of the type shown in Fig. 3, with a circular axis $\Gamma_{R}$ of radius $R$, a value of the radius $r=r_{1}$, and angular pitch $\theta_{p}=2 \pi / n$, where $n$ is equal to the number of turns of the helix (in Fig. 3, $n=5$ and the first coil begins and ends in the points $T$ and $T^{\prime}$, respectively).

2) The definition of another helix by a rotation $\Delta \theta<0$ of the previous helix around the axis $z-z$.

3) The definition of a third toroidal helix identical to the first one, but with a radius $r=r_{2}$ and $r_{2}>r_{1}$.

4) The definition of a fourth toroidal helix by another rotation again equals to $\Delta \theta<0$ of the helix with radius $r_{2}$ around the axis $z-z$.

Through this procedure, two pairs of identical and coaxial toroidal helices out of phase with the same angle $\Delta \theta<0$ are obtained. These helices represent the four edges of a helical toroidal magnet (see Fig. 4). The modulus of the magnetization vector $\mathbf{M}$ of this magnet is considered a priori constant. The direction of $\mathbf{M}$ is equal to that of the binormal unit vector $\hat{\mathbf{b}}$ relative to a fifth toroidal helix coaxial with those previously defined. This additional helix has a radius $r$ equal to the average radius $r_{m}=\left(r_{1}+r_{2}\right) / 2$ and is out of phase with an angle $\Delta \theta / 2<0$ with respect to the two helices generated in steps 1) and 3), respectively (see Fig. 4). The binormal unit vector $\hat{\mathbf{b}}$ is defined with reference to the respective normal $\hat{\mathbf{n}}$ and tangent $\hat{\mathbf{t}}$ unit vectors in the generic point $P$ that represents the common origin of the three unit vectors and $\mathbf{M}$. It is noticed that the unit vector $\hat{\mathbf{n}}$ has not the direction of the radius $r_{m}$.

\section{Regions Where the Field Is Evaluated}

In the computation subsequently developed, the regions, where the magnetic field is calculated, are defined by the successive positions of a flat surface of a movable element

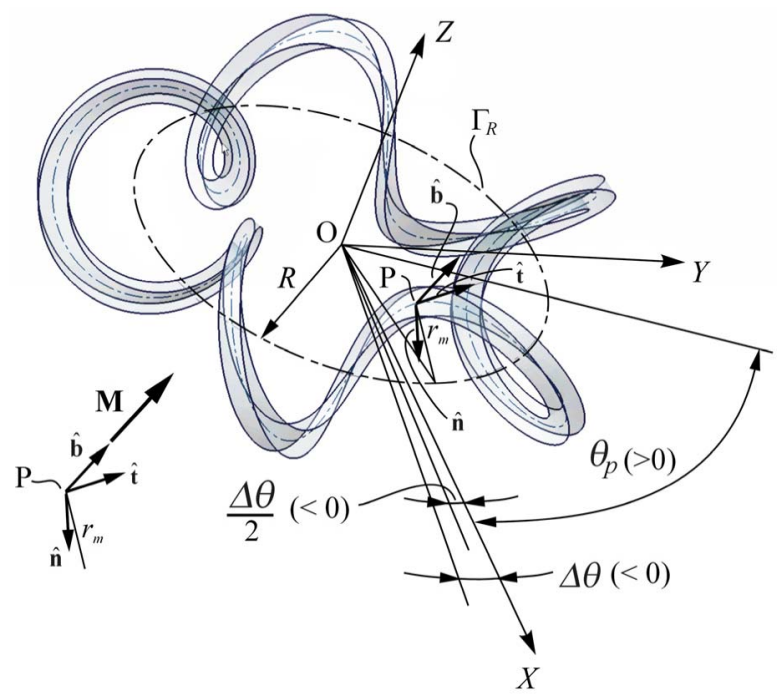

Fig. 4. Helical toroidal magnet and magnetization $\mathbf{M}$.

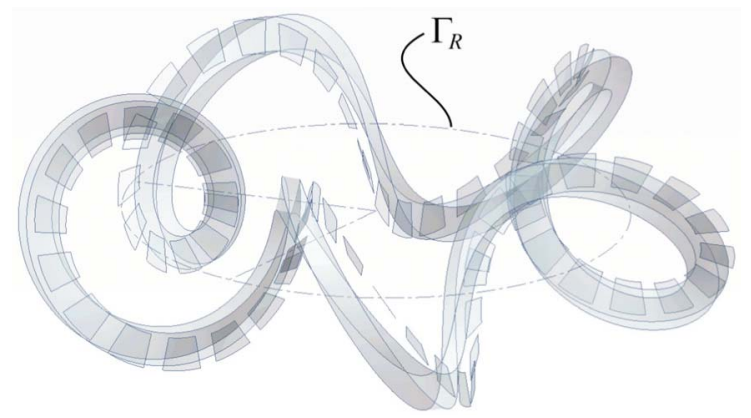

Fig. 5. Plane angular sectors where the magnetostatic field is evaluated.

animated by a helical toroidal motion. In Fig. 5, the sequence of such surfaces arranged frontally to the toroidal helicoidal magnet is illustrated. The number of these surfaces is equal to $n \times m$, where $n$ is the number of coils of the magnet and $m$ is the number of surfaces in correspondence with each coil. In Fig. 5, the positioning of each surface is quantitatively shown. These surfaces are circular sectors with inner and outer radius equal to the radii $r_{1}$ and $r_{2}$, respectively, as mentioned in Section II. The angle $\varphi$ defines the angle of the sector and its symmetry with respect to the mean radius $r_{m}$ previously mentioned. In Fig. 6, the final and initial point of this radius $r_{m}$ are denoted by $K_{m}$ and $U_{m}$, respectively. This radius describes the helical toroidal coil $\Gamma_{m}$, which is obtained by rotating with an angle $\Delta \theta_{m}>0$, and the toroidal helix $\Gamma$ around the $Z$-axis. The helix $\Gamma$ is the average one between the two helical toroidal edges that define the surface of the magnet placed in front of the angular sector. Also $\Gamma$ has a radius $r$ equal to $r_{m}$. The final point $K$ of this radius describes $\Gamma$ versus the angle $\theta_{U} . t$ is the tangent to $\Gamma$ in $K$, and $t_{m}$ is the corresponding tangent to $\Gamma_{m}$ in $K_{m}$. Therefore, $t$ and $t_{m}$ are not parallel. The angular sector belongs to the plane defined by the tangent $t_{m}$ and the point $U_{m}$ previously mentioned. In Fig. 7, the sector indicated in Fig. 6 is shown, but in this case, the same sector is in the first position denoted with 


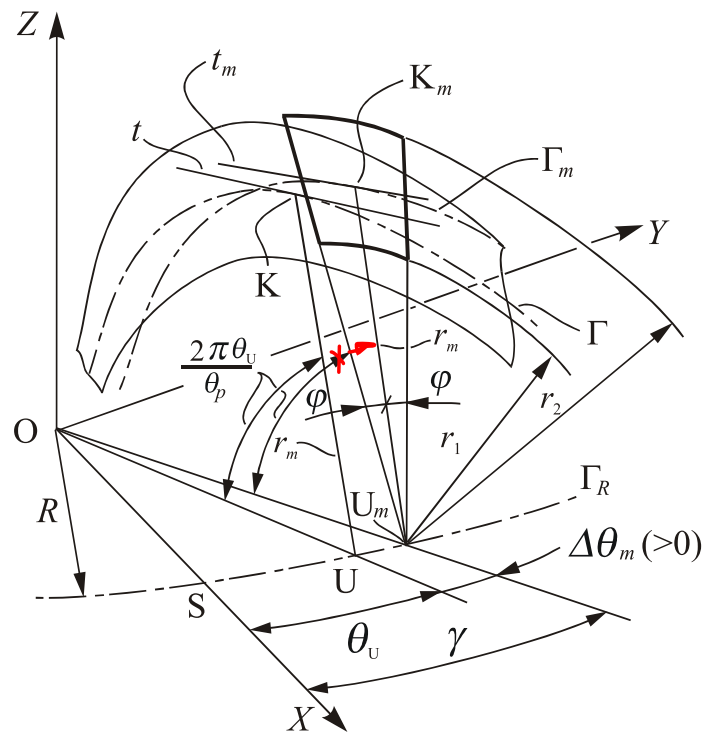

Fig. 6. Spatial localization of the generic angular plane sector.

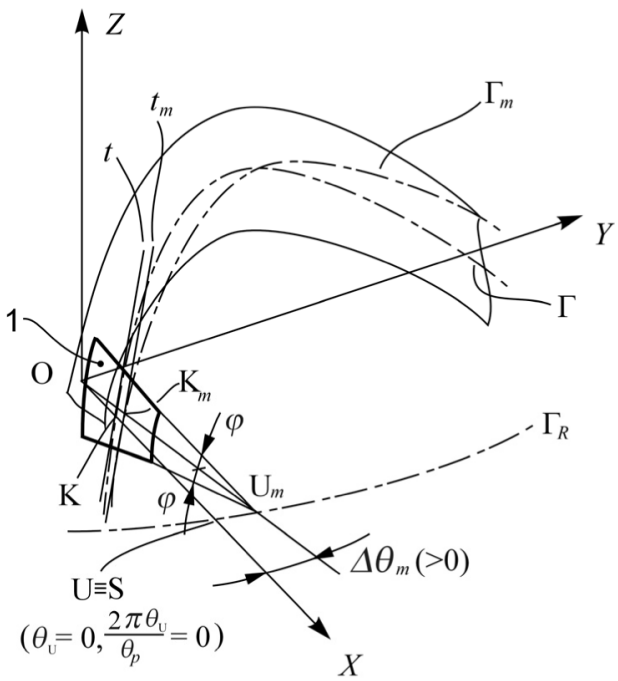

Fig. 7. Position of the first angular plane sector 1.

the index 1 . This position is defined by $\theta_{U}=0$. Consequently, the flat region of interest is positioned in such a way that its radius $r_{m}$ with extreme points $K_{m}$ and $U_{m}$ overlaps the horizontal segment $\mathrm{OU}_{m}=R$, which describes the circumference $\Gamma_{R}$. In relation to the geometry illustrated, for each coil of the magnet, we define 14 positions of the angular flat sector previously considered where the magnetic field will be calculated. Fig. 8 shows these 14 positions, equidistant from each other, with the point $K_{m}$ always belonging to the toroidal helix $\Gamma_{m}$.

The positioning of each region is always defined by the geometric constraints shown in Fig. 6. The equidistance among the regions is obtained by increasing the angle $\theta_{U}$ from zero to the value $\theta_{p}$ with a constant angular step. Since the calculation of the magnetic field can only be performed in fixed points, on the generic sector, an angular distribution of points where evaluation of the field is made is fixed. Fig. 9 shows the generic

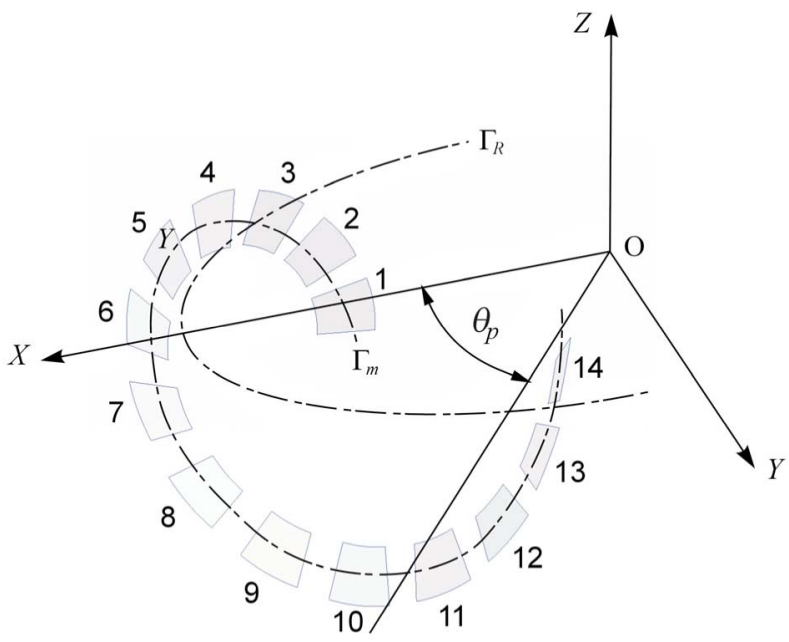

Fig. 8. Set of 14 angular plane sectors where the magnetostatic field is computed. Sectors positioned in front of the first coil of the magnet.

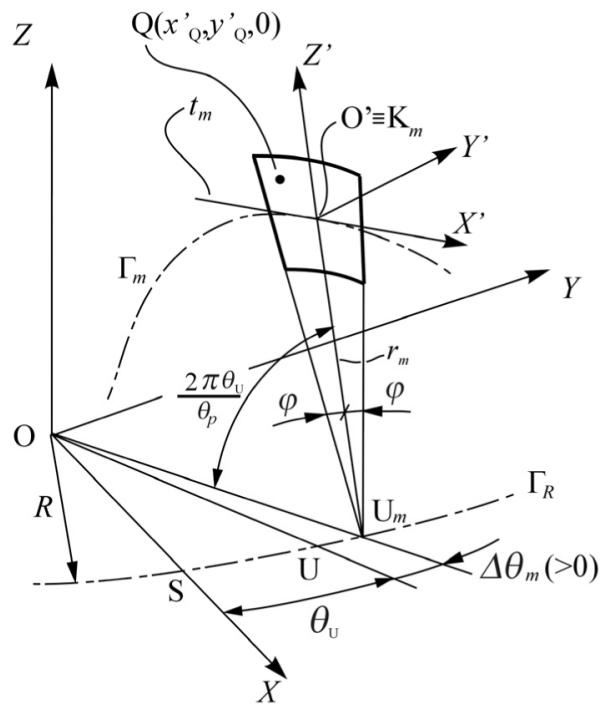

Fig. 9. System reference $O^{\prime}\left(X^{\prime}, Y^{\prime}, Z^{\prime}\right)$ integral to the generic angular plane sector.

point $Q$ with the coordinates $x_{Q}^{\prime}, y_{Q}^{\prime}$, and $z_{Q}^{\prime}=0$ relative to the $O^{\prime}\left(X^{\prime}, Y^{\prime}, Z^{\prime}\right)$ reference system integral with the generic sector. The origin $O^{\prime}$ of this reference system coincides with the point $K_{m}$ previously mentioned. The axes $X^{\prime}$ and $Z^{\prime}$ overlap the tangent $t_{m}$ and the radius $r_{m}$, respectively. The radius $r_{m}$ has extreme points $K_{m}$ and $U_{m}$.

\section{EQUATIONS FOR THE GEOMETRY DEFINITION OF THE SYSTEM}

The equations of the toroidal helix shown in Fig. 3 are

$$
\begin{aligned}
& x_{V}=\left[R-r \cos \left(\frac{2 \pi \theta}{\theta_{p}}\right)\right] \cos \theta \\
& y_{V}=\left[R-r \cos \left(\frac{2 \pi \theta}{\theta_{p}}\right)\right] \sin \theta \\
& z_{V}=r \sin \left(\frac{2 \pi \theta}{\theta_{p}}\right)
\end{aligned}
$$


where $x_{V}, y_{V}$, and $z_{V}$ represent the cartesian coordinates of the point $V$ that defines the helix versus the angle $\theta$. Substituting in these equations: 1) $r=r_{1}$; 2) $r=r_{2}$;3) $r=r_{1}$ with $\cos (\theta+\Delta \theta)$ in the place of $\cos \theta$ and $\sin (\theta+\Delta \theta)$ in the place of $\sin \theta$; and 4) $r=r_{2}$ with the same replacements of sines and cosines defined in 3 ), we correspondingly obtain the four helical toroidal edges that define the magnet (see Fig. 4). The mean toroidal helix of the magnet that we consider to define the tangent $\hat{\mathbf{t}}$, normal $\hat{\mathbf{n}}$, and binormal $\hat{\mathbf{b}}$ unit vectors, and also the magnetization $\mathbf{M}$ (Fig. 4) is again defined by (1)-(3). To obtain this helix, we have to substitute in the same equations $r=r_{m}$ and replace $\sin \theta$ with $\sin (\theta+\Delta \theta / 2)$ and $\cos \theta$ with $\cos (\theta+\Delta \theta / 2)$. The components $t_{x}, t_{y}$, and $t_{z}$ of the tangent versor $\hat{\mathbf{t}}$, with

$$
\beta=\theta+\frac{\Delta \theta}{2}
$$

are

$$
\begin{aligned}
& t_{x}(\beta)=\frac{x_{P}^{\prime}(\beta)}{\sqrt{x_{P}^{\prime}(\beta)^{2}+y_{P}^{\prime}(\beta)^{2}+z_{P}^{\prime}(\beta)^{2}}} \\
& t_{y}(\beta)=\frac{y_{P}^{\prime}(\beta)}{\sqrt{x_{P}^{\prime}(\beta)^{2}+y_{P}^{\prime}(\beta)^{2}+z_{P}^{\prime}(\beta)^{2}}} \\
& t_{z}(\beta)=\frac{z_{P}^{\prime}(\beta)}{\sqrt{x_{P}^{\prime}(\beta)^{2}+y_{P}^{\prime}(\beta)^{2}+z_{P}^{\prime}(\beta)^{2}}}
\end{aligned}
$$

where $x_{P}^{\prime}(\beta), y_{P}^{\prime}(\beta)$, and $z_{P}^{\prime}(\beta)$ represent the first derivatives with respect to $\theta$ of the coordinates $x_{P}(\beta), y_{P}(\beta)$, and $z_{P}(\beta)$ of the generic point $P$ that defines the average toroidal helix of the magnet (see Fig. 4). The components $n_{x}, n_{y}$, and $n_{z}$ of the unit normal vector $\hat{\mathbf{n}}$ are obtained versus the previous components $t_{x}, t_{y}$, and $t_{z}$ versus the relative first derivatives $t_{x}^{\prime}(\beta), t_{y}^{\prime}(\beta)$, and $t_{z}^{\prime}(\beta)$ with respect to $\beta$

$$
\begin{aligned}
& n_{x}(\beta)=\frac{t_{x}^{\prime}(\beta)}{\sqrt{t_{x}^{\prime}(\beta)^{2}+t_{y}^{\prime}(\beta)^{2}+t_{z}^{\prime}(\beta)^{2}}} \\
& n_{y}(\beta)=\frac{t_{y}^{\prime}(\beta)}{\sqrt{t_{x}^{\prime}(\beta)^{2}+t_{y}^{\prime}(\beta)^{2}+t_{z}^{\prime}(\beta)^{2}}} \\
& n_{z}(\beta)=\frac{t_{z}^{\prime}(\beta)}{\sqrt{t_{x}^{\prime}(\beta)^{2}+t_{y}^{\prime}(\beta)^{2}+t_{z}^{\prime}(\beta)^{2}}} .
\end{aligned}
$$

Regarding the binormal unit vector that fixes the direction of the magnetization $\mathbf{M}$, it is obtained by calculating the cross product of the versors $\hat{\mathbf{t}}$ and $\hat{\mathbf{n}}$

$$
\hat{\mathbf{b}}=\hat{\mathbf{t}} \wedge \hat{\mathbf{n}} .
$$

As soon as we substitute $r=r_{m}$ and replace $\sin \theta$ with $\sin \left(\theta_{U}+\Delta \theta_{m}\right)$ and $\cos \theta$ with $\cos \left(\theta_{U}+\Delta \theta_{m}\right)$ in (1)-(3), we obtain the parametric equations of the toroidal helix $\Gamma_{m}$ shown in Figs. 5-9. Now, if we let

$$
\gamma=\theta_{U}+\Delta \theta_{m}
$$

the coordinates $x_{K_{m}}(\gamma), y_{K_{m}}(\gamma)$, and $z_{K_{m}}(\gamma)$ of the generic point $K_{m}$ of $\Gamma_{m}$ can be evaluated. By calculating the tangent unit vector (not shown in any figure) in the point $K_{m}$ of the curve $\Gamma_{m}$, the tangent $t_{m}$ is achieved. The equations of the components of this unit vector have again the structure of (5)-(7) where, however, the derivatives $x_{P}^{\prime}(\beta)$, $y_{P}^{\prime}(\beta)$, and $z_{P}^{\prime}(\beta)$ are replaced with the derivatives $x_{K_{m}}^{\prime}(\gamma)$, $y_{K_{m}}^{\prime}(\gamma)$, and $z_{K_{m}}^{\prime}(\gamma)$ with respect to $\gamma$ of the coordinates $x_{K_{m}}(\gamma), y_{K_{m}}(\gamma)$, and $z_{K_{m}}(\gamma)$. In this way, the plane passing through $t_{m}$ and the point $U_{m}$ is defined. The generic angular sector where the magnetic field will be computed belongs to the plane passing through $t_{m}$ and $U_{m}$. As illustrated in Section III, this calculation will be performed only in a finite number of points belonging to the generic sector. With reference to the generic point $Q$, where the computation will be carried out, as soon as we fix its coordinates $x_{Q}^{\prime}, y_{Q}^{\prime}=0$, and $z_{Q}^{\prime}$ in the $O^{\prime}\left(X^{\prime}, Y^{\prime}, Z^{\prime}\right)$ orthogonal reference system (see Fig. 9) integral to the generic sector, it is advantageous to evaluate the coordinates of $Q$ with respect to the general $O(X, Y, Z)$ reference system. In order to compute these new coordinates $x_{Q}, y_{Q}$, and $z_{Q}$ that, as shown in the following section VI, will be directly used for evaluating the magnetic field, we consider the known formulas of coordinate transformation between two reference systems with origins that do not coincide. Therefore, we obtain

$$
\begin{aligned}
& x_{Q}=l_{1} x_{Q}^{\prime}+l_{2} y_{Q}^{\prime}+l_{3} x_{Q}^{\prime}+x_{O^{\prime}} \\
& y_{Q}=m_{1} x_{Q}^{\prime}+m_{2} y_{Q}^{\prime}+m_{3} x_{Q}^{\prime}+y_{O^{\prime}} \\
& z_{Q}=n_{1} x_{Q}^{\prime}+n_{2} y_{Q}^{\prime}+n_{3} x_{Q}^{\prime}+z_{O^{\prime}}
\end{aligned}
$$

where the terms $l_{1}, m_{1}, n_{1} ; l_{2}, m_{2}, n_{2}$; and $l_{3}, m_{3}, n_{3}$ are the direction cosines of the axes $X^{\prime}, Y^{\prime}, Z^{\prime}$ with respect to the axes $X, Y, Z$. $x_{O^{\prime}}, y_{O^{\prime}}$, and $z_{O^{\prime}}$ are the coordinates of the origin $O^{\prime}$ with respect to the $O(X, Y, Z)$ reference system. The direction cosines previously mentioned can be simply calculated by setting three points $P_{A}, P_{B}$, and $P_{C}$ on the respective axes $X^{\prime}, Y^{\prime}$, and $Z^{\prime}$. The coordinates of these points in the $O(X, Y, Z)$ system are $x_{A}(\gamma), y_{A}(\gamma), z_{A}(\gamma) ; x_{B}(\gamma)$, $y_{B}(\gamma), z_{B}(\gamma)$; and $x_{C}(\gamma), y_{C}(\gamma), z_{C}(\gamma)$. Now, if we let

$$
\begin{aligned}
& d_{A}(\gamma) \\
& =\sqrt{\left[x_{A}(\gamma)-x_{O^{\prime}}(\gamma)\right]^{2}+\left[y_{A}(\gamma)-y_{O^{\prime}}(\gamma)\right]^{2}+\left[z_{A}(\gamma)-z_{O^{\prime}}(\gamma)\right]^{2}}
\end{aligned}
$$

the direction cosines of the axis $x^{\prime}$ with respect the axes $x, y$, and $z$ are given by the following relations:

$$
\begin{aligned}
l_{1}(\gamma) & =\frac{x_{A}(\gamma)-x_{O^{\prime}}(\gamma)}{d_{A}(\gamma)} \\
m_{1}(\gamma) & =\frac{y_{A}(\gamma)-y_{O^{\prime}}(\gamma)}{d_{A}(\gamma)} \\
n_{1}(\gamma) & =\frac{z_{A}(\gamma)-z_{O^{\prime}}(\gamma)}{d_{A}(\gamma)} .
\end{aligned}
$$

Similarly, for the axes $y^{\prime}$ and $z^{\prime}$, the direction cosines are equal to

$$
\begin{aligned}
l_{2}(\gamma) & =\frac{x_{B}(\gamma)-x_{O^{\prime}}(\gamma)}{d_{B}(\gamma)} \\
m_{2}(\gamma) & =\frac{y_{B}(\gamma)-y_{O^{\prime}}(\gamma)}{d_{B}(\gamma)} \\
n_{2}(\gamma) & =\frac{z_{B}(\gamma)-z_{O^{\prime}}(\gamma)}{d_{B}(\gamma)}
\end{aligned}
$$




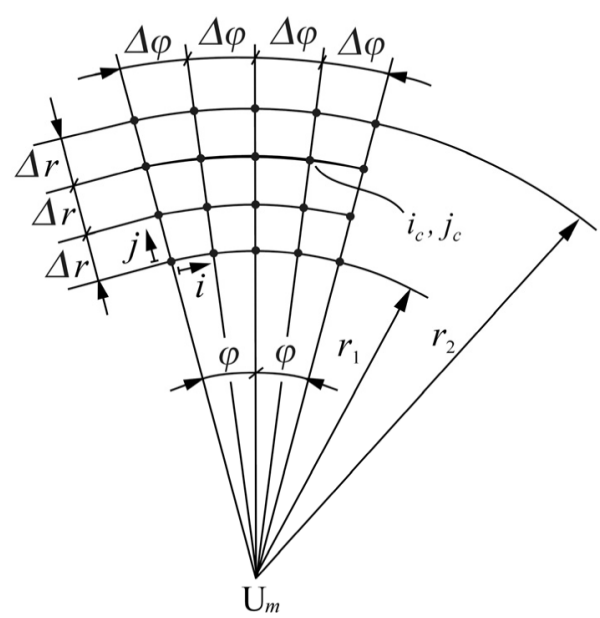

Fig. 10. Identification of the points belonging to the generic angular plane sector where the magnetostatic field is computed.

and

$$
\begin{aligned}
l_{3}(\gamma) & =\frac{x_{C}(\gamma)-x_{O^{\prime}}(\gamma)}{d_{C}(\gamma)} \\
m_{3}(\gamma) & =\frac{y_{C}(\gamma)-y_{O^{\prime}}(\gamma)}{d_{C}(\gamma)} \\
n_{3}(\gamma) & =\frac{z_{C}(\gamma)-z_{O^{\prime}}(\gamma)}{d_{C}(\gamma)}
\end{aligned}
$$

respectively, where

$$
\begin{aligned}
& d_{B}(\gamma) \\
& =\sqrt{\left[x_{B}(\gamma)-x_{O^{\prime}}(\gamma)\right]^{2}+\left[y_{B}(\gamma)-y_{O^{\prime}}(\gamma)\right]^{2}+\left[z_{B}(\gamma)-z_{O^{\prime}}(\gamma)\right]^{2}}
\end{aligned}
$$

and

$$
\begin{aligned}
& d_{C}(\gamma) \\
& =\sqrt{\left[x_{C}(\gamma)-x_{O^{\prime}}(\gamma)\right]^{2}+\left[y_{C}(\gamma)-y_{O^{\prime}}(\gamma)\right]^{2}+\left[z_{C}(\gamma)-z_{O^{\prime}}(\gamma)\right]^{2}} .
\end{aligned}
$$

\section{Points And Angular Sectors WHERE THE FIELD IS COMPUTED}

The calculation of the magnetic field on each angular sector has been performed in correspondence with two arrays of 20 points. The first matrix defines 20 points uniformly distributed on the same sector, as shown in Fig. 10. The generic point is identified by the pair of indices $\left(i_{c}, j_{c}\right)$. The points $\left(i_{c}, j_{c}\right)$ are placed on concentric circular arcs, whose centre is the point $U_{m}$. In this first case, the field was calculated in $20 \times 14 \times 5=1400$ points by considering all the 14 sectors and five coils. As shown in the following section VII, the field values evaluated in these points highlight its axial symmetry. The second field evaluation was carried out in 625 points of each angular sector. In this case, it is $i_{c}=1,2, \ldots, 25$ and $j_{c}=1,2, \ldots, 25(625=25 \times 25)$. These points are always uniformly distributed on the angular sector in a way similar to that illustrated in Fig. 10. However, in this analysis, the field was calculated only relatively to the 14 sectors of a single coil, the first one, in a number of points equal to $14 \times 625=8750$.
TABLE I

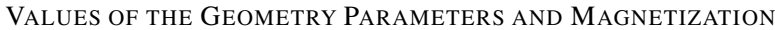
Modulus $|\mathbf{M}|$ OF THE MAGNet

\begin{tabular}{|c|c|c|c|c|c|c|c|}
\hline $\begin{array}{c}n \\
(\mathrm{coils})\end{array}$ & $\theta_{p}(\mathrm{rad})$ & $\Delta \theta(\mathrm{rad})$ & $\begin{array}{c}R \\
(\mathrm{~mm})\end{array}$ & $\begin{array}{c}r_{1} \\
(\mathrm{~mm})\end{array}$ & $\begin{array}{c}r_{2} \\
(\mathrm{~mm})\end{array}$ & $\begin{array}{c}r_{m} \\
(\mathrm{~mm})\end{array}$ & $|\mathbf{M}|(\mathrm{A} / \mathrm{m})$ \\
\hline 5 & $72.0 \times \pi / 180$ & $-5.0 \times \pi / 180$ & 220 & 67.5 & 97.5 & 82.5 & $4.3 \times 10^{5}$ \\
\hline
\end{tabular}

As a matter of fact, in relation to the axial symmetry of the field, the field values in the other $8750 \times 4=35000$ points relating to the sectors positioned in front of the other four coils can be obtained from the values evaluated in the previous 8750 points without any calculation of integration. To obtain these values, it is sufficient to rotate around the $Z$-axis, and the components along $X$ and $Y$ of the field calculated in the 8750 points. The rotation angle must be equal to the angle that defines the angular positioning of the each coil.

\section{Magnetostatic Field Evaluation}

In the previous sections IV and V, the geometry of the case magnet study has been described. All the parametric equations of the curves and the coordinates of the points were obtained. With reference to Fig. 3 and (1)-(3), by fixing the values of $n, \theta_{p}, R, r=r_{1}, r=r_{2}$, and $\Delta \theta$ indicated in Table I, we achieved the four toroidal helicies that define the edges of the magnet. The fifth toroidal helix that represents the axis of the magnet, on which the tangent $\hat{\mathbf{t}}$, normal $\hat{\mathbf{n}}$, and binormal $\hat{\mathbf{b}}$ unit vectors have been considered (see Fig. 4), is again obtained by (1)-(3) by setting $r=r_{m}$. The value of $r_{m}$ and the modulus of the magnetization vector $\mathbf{M}$ are reported in Table I. In relation to the regions, where the field has been calculated, Table II shows the values of the geometric parameters $\Delta \theta_{m}$, $\theta_{U}, \varphi$, and $\gamma$ relative to the 14 angular plane sectors frontally positioned to the first coil [see Figs. 6-8 and (12)]. The value of $\varphi$ in Table II has been fixed to obtain an angular flat sector neither too large neither too narrow. If the angular flat sector is too large ( $\varphi$ has a high value), the 14 sectors facing each magnet coil are partially placed one upon another and the graphical representation is not clear (Figs. 5, 9, and 11 became very confused and it is difficult to check the correctness of the system geometry). Moreover, an angular sector too large can penetrate the surface of the helical toroidal magnet facing the same sector (in this case, the air gap is negative, and it is not a real configuration). In relation to this problem, the correct value of $\varphi$ also depends on the value of $\Delta \theta_{m}$ that was fixed equal to $4^{\circ}$ (see Table II). If the angular flat sector is too narrow ( $\varphi$ has a low value), the sets of points, where we calculate the field, are too far one in respect to the other: a possible careful spline interpolation of the field values between two adjacent positions of two angular flat sectors where the same field is really calculated will not be possible. In relation to the general sizing of the device study, a good value of $\varphi$ is equal to $10^{\circ}$ : by a $3-\mathrm{D}$ CAD simulation, we have checked that this value of $\varphi$ (with reference to $\Delta \theta_{m}=4^{\circ}$ ) is suitable to avoid penetration and to obtain a correct distance between an angular flat sector and the following one. Two sets of simulations have been performed. The first set concerns the evaluation of the 
TABLE II

Values of the Geometry Parameters of the Angular Plane Sectors Where the Magnetostatic Field Is Computed

\begin{tabular}{|c|c|c|c|c|c|c|c|c|}
\hline$u$ & \multicolumn{7}{|c|}{$\Delta \theta_{m}=4 \times \pi / 180 \mathrm{rad}(=4$ degrees $), \quad \varphi=10 \times \pi / 180 \mathrm{rad}(=10$ degrees $)$} \\
\hline \multirow{3}{*}{1} & $n_{\text {sec }}$ & 1 & 2 & 3 & 4 & 5 & 6 & 7 \\
\cline { 2 - 9 } & $\theta_{\mathrm{U}(\mathrm{rad})}$ & $0.0 \times \pi / 180$ & $5.4 \times \pi / 180$ & $10.8 \times \pi / 180$ & $16.2 \times \pi / 180$ & $21.6 \times \pi / 180$ & $27.0 \times \pi / 180$ & $32.4 \times \pi / 180$ \\
\cline { 2 - 9 } & $\gamma(\mathrm{rad})$ & $4.0 \times \pi / 180$ & $9.4 \times \pi / 180$ & $14.8 \times \pi / 180$ & $20.2 \times \pi / 180$ & $24.6 \times \pi / 180$ & $31.0 \times \pi / 180$ & $36.4 \times \pi / 180$ \\
\cline { 2 - 9 } & $n_{\text {sec }}$ & 8 & 9 & 10 & 11 & 12 & 13 & 14 \\
\cline { 2 - 9 } & $\theta_{\mathrm{U}(\mathrm{rad})}$ & $37.8 \times \pi / 180$ & $43.2 \times \pi / 180$ & $48.6 \times \pi / 180$ & $54.0 \times \pi / 180$ & $59.4 \times \pi / 180$ & $64.8 \times \pi / 180$ & $70.2 \times \pi / 180$ \\
\cline { 2 - 9 } & $\gamma(\mathrm{rad})$ & $41.8 \times \pi / 180$ & $47.2 \times \pi / 180$ & $56.2 \times \pi / 180$ & $58.0 \times \pi / 180$ & $63.4 \times \pi / 180$ & $68.8 \times \pi / 180$ & $74.2 \times \pi / 180$ \\
\hline
\end{tabular}

TABLE III

Parameters of the Points Matrix Where the Field Is Computed

\begin{tabular}{|c|c|c|}
\hline Simulation $n$. & $\Delta \varphi(\mathrm{rad})$ & $\Delta r(\mathrm{~mm})$ \\
\hline 1 & $5.0 \times \pi / 180$ & 10 \\
\hline 2 & $(20.0 / 24) \times \pi / 180$ & $30.0 / 24$ \\
\hline
\end{tabular}

field in all the $14 \times 5=70$ flat regions shown in Fig. 5 . In general, by fixing $\theta_{U}$ by the subsequent relationship

$$
\theta_{U}=\left[72.0(u-1)+\frac{70.2}{13}\left(n_{\mathrm{sec}}-1\right)\right] \frac{\pi}{180}
$$

the quantitative location of these regions is obtained. In (28), $u=1,2, \ldots, n(=5)$ and $n_{\mathrm{sec}}=1,2, \ldots, 14$ represent the current coil and the number of the plane sector positioned in front of the same coil, respectively (see Fig. 8). Substituting $u=1$ in (28), we obtain the values shown in Table II. By fixing $u>1$, we simply rotates, for $72^{\circ}$, the set of 14 plane sectors around the $Z$-axis. Therefore, as soon as $\theta_{U}$ is known, it is possible to achieve the values of the angle $2 \pi \theta_{U} / \theta_{p}$ that fixes the angular position of the generic flat sector. This sector belongs to the plane identified by the tangent $t_{m}$ and the radius $r_{m}$ with the initial and the final points $U_{m}$ and $K_{m}$, respectively (see Figs. 6 and 7). With reference again to this first set of evaluations, on each of the 70 plane sectors, a matrix constituted by 20 points was considered (see Fig. 10). The values of $\Delta r$ and $\Delta \varphi$ that define the position of such points in correspondence to $i_{c}=1,2, \ldots, 5$ and $j_{c}=1,2, \ldots, 4$ have been reported in Table III. In the case of the second set of simulations, where the field is calculated with reference only to the first coil (see the values of $\theta_{U}$ reported in Table I), $25 \times 25=625\left(i_{c}=1,2, \ldots, 25\right.$ and $\left.j_{c}=1,2, \ldots, 25\right)$ points were considered. For these simulations, the values of $\Delta r$ and $\Delta \varphi$ that define the position of the 625 points belonging to each one of the 14 sectors positioned in front of the first coil have also been indicated in Table III. In relation to the $\theta_{U}$ values, it is observed that this angle varies from $0^{\circ}$ to $70.2^{\circ}$. Consequently, the minimum and the maximum values of $\gamma$ are equal to $4^{\circ}$ and $74.2^{\circ}$, respectively. This choice implies that the angular plane sector $n .14$ where we compute the field (see Fig. 8) is not positioned as the sector $n .1$ (see Fig. 7). Sector $n .14$ is placed in such a way to partially overlap the next sector $n$. 1 relative to the second set of 14 sectors positioned in front of the second coil of the magnet. This arrangement of sectors enables us to distinguish the sector $n .14$ associated with the $i$ th coil from the sector $n .1$ relative to the $i+1$ th coil. If one assumes a distribution of 14 sectors so that the sector $n .14$ is overlapped with the sector $n .1$ (in such a case, the increase of $\theta_{U}$ is not equal to $5.4^{\circ}$, but is equal to $72 / 13=5.54^{\circ}$ ) the sectors $n .14$ and $n$. 1 positioned in front of the coils $i$ th and $i+1$ th, respectively, are no longer identifiable: the two sectors overlap perfectly. In order to visually check the correctness of the areas where we actually calculate the field, this distinction must be clear, therefore the choice (however, not the only one possible) to vary $\theta_{U}$ by a step equal to $5.4^{\circ}$ is justified. For example, Fig. 11 shows the position of the two sectors $n .14$ and $n .1$ in relation to the case of the coils $i=1, i+1=2$, and step of $\theta_{U}$ equal to $5.4^{\circ}$ (see Table I). The calculation of the magnetic field in all the points indicated in Section $\mathrm{V}$ has been carried out using the method of the magnetic charges [1]-[5]. This method considers the integration of the Maxwell equations

$$
\begin{aligned}
\nabla \times \mathbf{H} & =0 \\
\nabla \cdot \mathbf{B} & =0
\end{aligned}
$$

where $\mathbf{H}$ and $\mathbf{B}$ represent the magnetic field strength and the magnetic flux density, respectively. Contrary to the finiteelement analysis [13], [32] and also new methods (for example, the natural element method [33]), the magnetic charges approach considers the integration of (29) and (30) only with reference to the specific regions of interest. In this way, accuracy and time computations can be highly increased and reduced, respectively. By considering the $O(X, Y, Z)$ reference system, the method of the magnetic charges defines the so-called volume charge density $\rho_{M}(\mathbf{P})$ and surface charge density $\sigma_{M}(\mathbf{P})$ relative to the generic point $P$ of the magnet

$$
\begin{aligned}
& \rho_{M}(\mathbf{P}) \equiv-\nabla \cdot \mathbf{M}(\mathbf{P}) \\
& \sigma_{M}(\mathbf{P}) \equiv \mathbf{M}(\mathbf{P}) \cdot \hat{\mathbf{n}} .
\end{aligned}
$$

In (31) and (32), $\mathbf{P}$ is the vector, whose extremes are the origin of the reference system previously mentioned and the point $P$. In this context, it is known [1], [2], [34] that the magnetic flux density $\mathbf{B}$ in a generic point $P^{\prime}$ is given by the sum of two terms $\mathbf{B}_{V}\left(\mathbf{P}^{\prime}\right)$ and $\mathbf{B}_{S}\left(\mathbf{P}^{\prime}\right)$ associated with $\rho_{M}(\mathbf{P})$ and $\sigma_{M}(\mathbf{P})$, respectively

$$
\mathbf{B}\left(\mathbf{P}^{\prime}\right)=\mathbf{B}_{V}\left(\mathbf{P}^{\prime}\right)+\mathbf{B}_{S}\left(\mathbf{P}^{\prime}\right)
$$

where $\mathbf{P}^{\prime}$ represents the vector whose origin coincides with that of the $O(X, Y, Z)$ reference system. The final point 


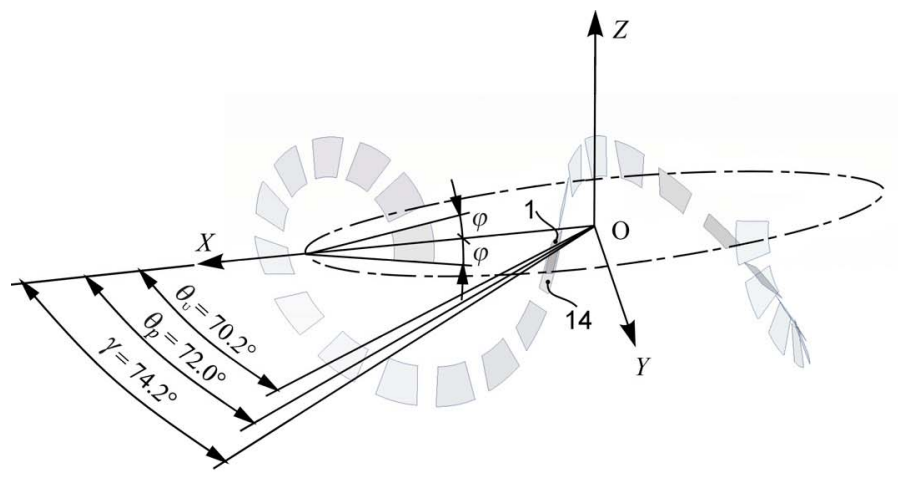

(a)

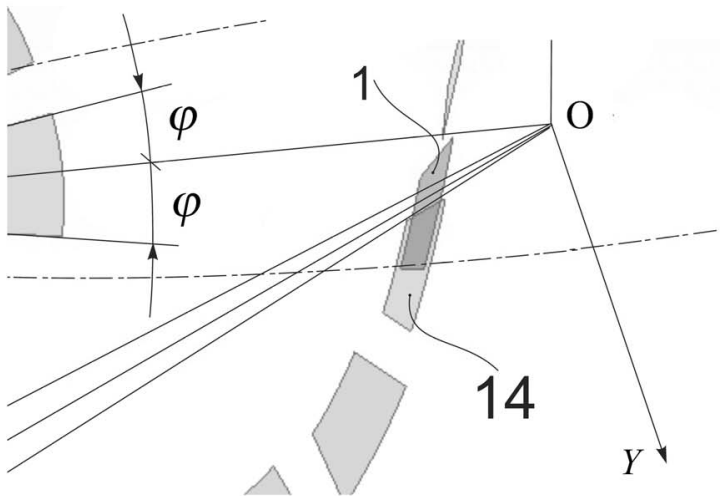

(b)

Fig. 11. (a) First and second set of plane angular sectors where the magnetic flux density has been computed. (b) Detail of the partial superimposition between the last sector (14) of the first set and the first sector (1) of the second set.

of $\mathbf{P}^{\prime}$ is $P^{\prime} . \mathbf{B}_{V}\left(\mathbf{P}^{\prime}\right)$ and $\mathbf{B}_{S}\left(\mathbf{P}^{\prime}\right)$ are given by the following expressions [34]:

$$
\mathbf{B}_{V}\left(\mathbf{P}^{\prime}\right)=\frac{\mu_{0}}{4 \pi} \int_{V} \frac{\rho_{M}(\mathbf{P})\left(\mathbf{P}^{\prime}-\mathbf{P}\right)}{\left|\mathbf{P}^{\prime}-\mathbf{P}\right|^{3}} d V
$$

and

$$
\mathbf{B}_{S}\left(\mathbf{P}^{\prime}\right)=\frac{\mu_{0}}{4 \pi} \int_{S} \frac{\sigma_{M}(\mathbf{P})\left(\mathbf{P}^{\prime}-\mathbf{P}\right)}{\left|\mathbf{P}^{\prime}-\mathbf{P}\right|^{3}} d S
$$

where $\left|\mathbf{P}^{\prime}-\mathbf{P}\right|$ is the modulus of the vector $\mathbf{P}^{\prime}-\mathbf{P}, S$ is the surface enclosing the volume $V$ of the magnet, and $\mu_{0}$ is the free-space permeability. In order to simplify the calculation, the field produced by the magnet has been obtained by considering only the contribution $\mathbf{B}_{S}\left(\mathbf{P}^{\prime}\right)$ of the surface charge density $\sigma_{M}(\mathbf{P})$. In relation to this simplification, it is noticed that, with reference to the helicoidal magnets with the straight axis and magnetization vector $\mathbf{M}$ arranged in a similar manner to that shown in Fig. 4, the volume charge density $\rho_{M}(\mathbf{P})$ is null [3]. Conversely, if the axis of the magnet is curved, the contribution of $\rho_{M}(\mathbf{P})$ increases versus the radius of curvature of the magnet axis. In order to evaluate the effect of $\rho_{M}(\mathbf{P})$, it is necessary to use (31) and (34), but surely both $\mathbf{B}_{V}\left(\mathbf{P}^{\prime}\right)$ and $\mathbf{B}_{S}\left(\mathbf{P}^{\prime}\right)$ are characterized by the same type of axial symmetry. In this regard, let us consider a rotation of the point $P^{\prime}$ for an angle equal to the angular pitch of the coils of the magnet around the $Z$-axis. It is observed that the set of all the infinite vectors $\mathbf{P}^{\prime}-\mathbf{P}$ that define the distance of $P^{\prime}$ from the corresponding infinitesimal volumes $d V$ and areas $d S$ relative to the neighborhood of the same point $P^{\prime}$, change only their directions, while the moduli keep constant values. Moreover, $\mathbf{P}$ is also independent of the rotation; therefore, $\rho_{M}(\mathbf{P})$ and $\sigma_{M}(\mathbf{P})$ are also independent of the new position of $P^{\prime}$. It follows that $\mathbf{B}_{V}\left(\mathbf{P}^{\prime}\right)$ and $\mathbf{B}_{S}\left(\mathbf{P}^{\prime}\right)$ keep their moduli constant when $P^{\prime}$ rotates around $Z$. In any case, the rotation of $P^{\prime}$ defines the new directions of the two magnetic flux densities. Thus, the components of $\mathbf{B}_{V}\left(\mathbf{P}^{\prime}\right)$ and $\mathbf{B}_{S}\left(\mathbf{P}^{\prime}\right)$ along the axes $X$ and $Y$ vary without changing the relative modules $\left|\mathbf{B}_{V}\left(\mathbf{P}^{\prime}\right)\right|$ and $\left|\mathbf{B}_{S}\left(\mathbf{P}^{\prime}\right)\right|$. In confirmation of these considerations, we can consider an observer in a generic point of any plane sector relative to a coil. For example, if we choose the first coil and the first plane sector (see the sector $n .1$ in Fig. 8) and the point $P^{\prime}$ defined by $i_{c}=1$ and $j_{c}=1$ (see Fig. 10) where the field has to be computed, the observer will detect the following contributions of magnetic flux densities in point $P^{\prime}$ :

$$
d \mathbf{B}_{V}\left(\mathbf{P}^{\prime}\right)=\frac{\mu_{0}}{4 \pi} \frac{\rho_{M}(\mathbf{P})\left(\mathbf{P}^{\prime}-\mathbf{P}\right)}{\left|\mathbf{P}^{\prime}-\mathbf{P}\right|^{3}} d V
$$

and

$$
d \mathbf{B}_{S}\left(\mathbf{P}^{\prime}\right)=\frac{\mu_{0}}{4 \pi} \frac{\sigma_{M}(\mathbf{P})\left(\mathbf{P}^{\prime}-\mathbf{P}\right)}{\left|\mathbf{P}^{\prime}-\mathbf{P}\right|^{3}} d S .
$$

These contributions are evaluated by the observer in $P^{\prime}$ which watches all the infinitesimal volumes $d V$ and areas $d S$ distributions that constitute the magnet. If the observer moves to the new point $P^{\prime}$, again identified by $i_{c}=1$ and $j_{c}=1$ but belonging to the sector $n$. 1 relative to the second coil, he sees the same distributions of volumes $d V$ and areas $d S$. Thus, from the point of view of the same observer, in the passage from the point $i_{c}=1$ and $j_{c}=1$ relative to the first coil to the new point $i_{c}=1$ and $j_{c}=1$ relative to the second coil, nothing has changed, i.e., the observer always detects the same magnet, exactly if there had been no rotation. It follows that before and after the rotation, the same observer, from his point of view, notices in $P^{\prime}$ always the same magnetic flux densities $\mathbf{B}_{V}\left(\mathbf{P}^{\prime}\right)$ and $\mathbf{B}_{S}\left(\mathbf{P}^{\prime}\right)$. Conversely, an observer placed in the absolute $O(X, Y, Z)$ reference system, after the rotation certainly detects that $\mathbf{B}_{V}\left(\mathbf{P}^{\prime}\right)$ and $\mathbf{B}_{S}\left(\mathbf{P}^{\prime}\right)$ have rotated around the axis $Z$. Therefore, the components of these two magnetic flux densities along $X$ and $Y$ are changed. However, this change must ensure that the observer integral with the point $P^{\prime}$, before and after the rotation around $Z$, does not detect any change in the magnetic flux densities (for this observer, the displacement from the original position $P^{\prime}$ to the new position $P^{\prime}$ rotated has not changed the magnet). Consequently, this condition can be satisfied only if, after the rotation of $P^{\prime}$, the new components of $\mathbf{B}_{V}\left(\mathbf{P}^{\prime}\right)$ and $\mathbf{B}_{S}\left(\mathbf{P}^{\prime}\right)$ in the absolute $O(X, Y, Z)$ reference system, ensure that the moduli $\left|\mathbf{B}_{V}\left(\mathbf{P}^{\prime}\right)\right|$ and $\left|\mathbf{B}_{S}\left(\mathbf{P}^{\prime}\right)\right|$ do not vary. Figs. 12 and 13 graphically show the situation described above. In these figures, $P^{\prime}$ and $P^{\prime \prime}$ represent the 


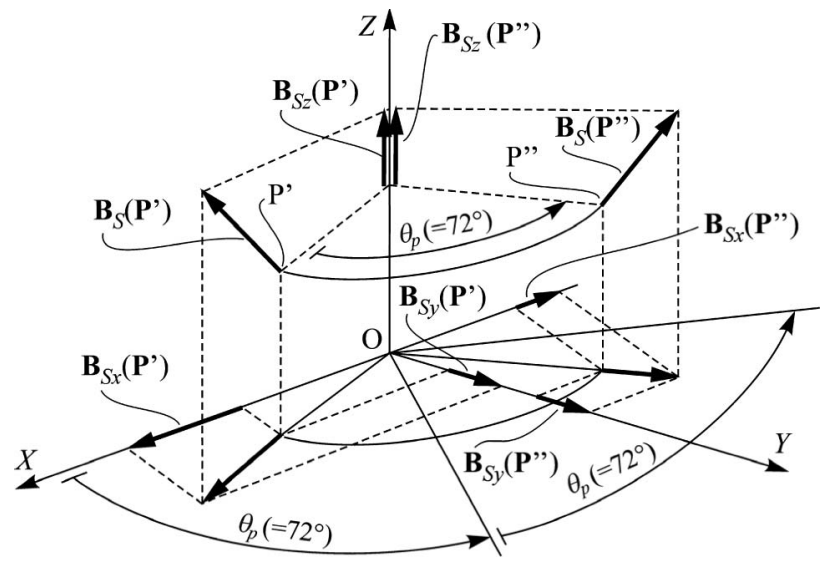

Fig. 12. Magnetic flux density $\mathbf{B}_{S}\left(\mathbf{P}^{\prime}\right)$ rotated for $72^{\circ}$.

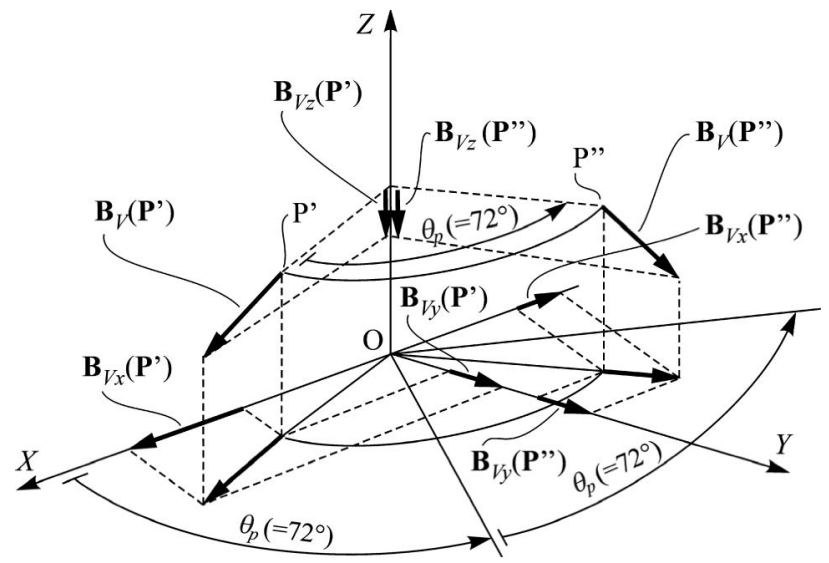

Fig. 13. Magnetic flux density $\mathbf{B}_{\boldsymbol{V}}\left(\mathbf{P}^{\prime}\right)$ rotated for $72^{\circ}$.

initial and the final position, respectively, of the point where we consider the field before and after a rotation equal to $72^{\circ}$ of the same point around the axis $Z$. The previous reasoning can be applied to all the points $P^{\prime}$ of each plane sector positioned in front of each coil. Consequently, the axial symmetry of the field generated by the helicoidal toroidal magnet is justified. Now, we can also denote by the term homologous points, all the points $P^{\prime}$ associated with the rotations identified by $k \theta_{p}(k=1,2, \ldots, 5)$ and related to the angular pitch of the five coils of the system. In Section VII, the numerical results which confirm the above considerations will be illustrated. In relation to the field calculation, the same simplifying assumptions considered in [31] have been used. These simplifications concern the evaluation of the surface charge density given by (32). In this regard, it is observed that four magnetic surface charge densities $\sigma_{M 1}(\mathbf{P}), \sigma_{M 2}(\mathbf{P}), \sigma_{M 3}(\mathbf{P})$, and $\sigma_{M 4}(\mathbf{P})$ have to be considered. These quantities are always evaluated by (32). Each of them is associated with the respective helical toroidal surfaces $S_{1}, S_{2}, S_{3}$, and $S_{4}$ that define the volume of the magnet (see Fig. 14). By considering the normal versors $\hat{\mathbf{n}}_{S 1}, \hat{\mathbf{n}}_{S 2}, \hat{\mathbf{n}}_{S 3}$, and $\hat{\mathbf{n}}_{S 4}$ in the generic points $P$ of such areas, we notice that $\hat{\mathbf{n}}_{S 1}$ and $\hat{\mathbf{n}}_{S 2}$ are approximately parallel to the magnetization vector $\mathbf{M}$ fixed (see Fig. 4), while $\hat{\mathbf{n}}_{S 3}$ and $\hat{\mathbf{n}}_{S 4}$ are nearly perpendicular to $\mathbf{M}$. Therefore, in accordance

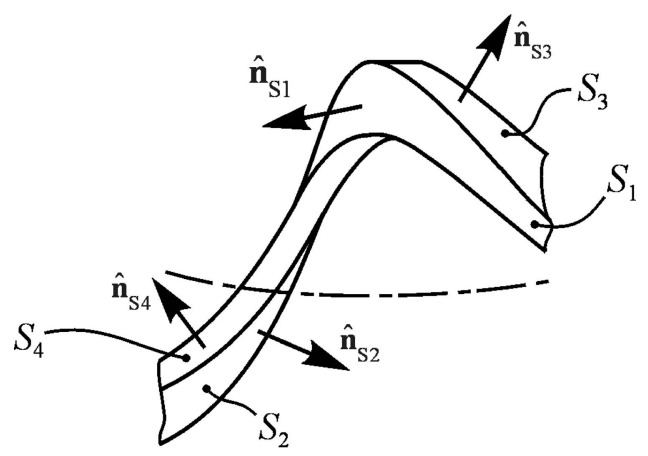

Fig. 14. Unit normal vectors relative to the surfaces of the magnet.

with (32), it results that only

$\sigma_{M 1}(\mathbf{P}) \cong M_{x}(\mathbf{P}) n_{S 1 x}(\mathbf{P})+M_{y}(\mathbf{P}) n_{S 1 y}(\mathbf{P})+M_{z}(\mathbf{P}) n_{S 1 z}(\mathbf{P})$

and

$\sigma_{M 2}(\mathbf{P}) \cong M_{x}(\mathbf{P}) n_{S 2 x}(\mathbf{P})+M_{y}(\mathbf{P}) n_{S 2 y}(\mathbf{P})+M_{z}(\mathbf{P}) n_{S 2 z}(\mathbf{P})$

are different from zero, while

$$
\sigma_{M 3}(\mathbf{P}) \cong 0
$$

and

$$
\sigma_{M 4}(\mathbf{P}) \cong 0
$$

Consequently, we obtain

$$
\mathbf{B}_{S}\left(\mathbf{P}^{\prime}\right) \cong \mathbf{B}_{S 1}\left(\mathbf{P}^{\prime}\right)+\mathbf{B}_{S 2}\left(\mathbf{P}^{\prime}\right)
$$

where using (35)

$$
\mathbf{B}_{S 1}\left(\mathbf{P}^{\prime}\right)=\frac{\mu_{0}}{4 \pi} \int_{S 1} \frac{\sigma_{M 1}(\mathbf{P})\left(\mathbf{P}^{\prime}-\mathbf{P}\right)}{\left|\mathbf{P}^{\prime}-\mathbf{P}\right|^{3}} d S
$$

and

$$
\mathbf{B}_{S 2}\left(\mathbf{P}^{\prime}\right)=\frac{\mu_{0}}{4 \pi} \int_{S 2} \frac{\sigma_{M 2}(\mathbf{P})\left(\mathbf{P}^{\prime}-\mathbf{P}\right)}{\left|\mathbf{P}^{\prime}-\mathbf{P}\right|^{3}} d S .
$$

\section{Numerical Evaluation OF THE FiELd SyMmetry}

The axial symmetry of the magnetostatic field generated by the magnet has been shown by two checks.

1) The calculation of the absolute value of the maximum percentage difference found between the moduli of the field relative to all the pairs of homologous points where the field is evaluated.

2) Verifying that the components of the field along the axes of a reference system integral to each group of plane sectors relating to a given magnet coil do not change when we consider a different magnet coil.

This second check was carried out by calculating all three components of the field in all the homologous points previously mentioned. The vector of the magnetic flux density is obtained by the calculation described in Section VI (i.e., the vector of the magnetic flux density is defined by the components evaluated with respect to the $O(X, Y, Z)$ reference system). 
TABLE IV

PAIRs OF COILS FOR Homologous PoINTS DEFINITION

\begin{tabular}{|l|l|l|l|l|l|l|l|l|l|l|}
\hline \multicolumn{10}{|c|}{$C_{n, k}=C_{5,2}=10$} \\
\hline Coil $s$ & 1 & 1 & 1 & 1 & 2 & 2 & 2 & 3 & 3 & 4 \\
Coil $t$ & 2 & 3 & 4 & 5 & 3 & 4 & 5 & 4 & 5 & 5 \\
\hline
\end{tabular}

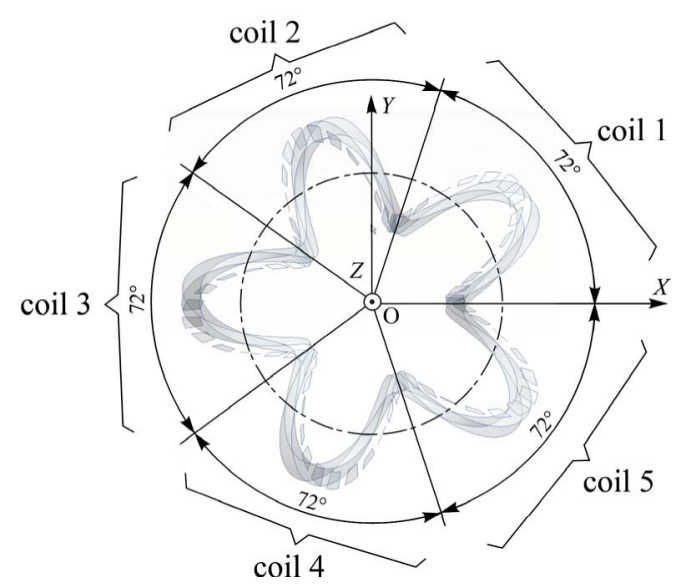

Fig. 15. Helicoidal toroidal magnet with five coils and relative plane angular sectors where the magnetostatic filed is evaluated.

\section{A. Computation of the Maximum Percentage Difference Between the Moduli of the Field in Homologous Points}

In order to perform this computation, we evaluate all the combinations of coil pairs that can be obtained from the five coils that constitute the toroidal helical magnet. In general, denoted by $C_{n, k}$, the number of nonsorted subsets of $k$ distinct elements of a set of $n$ elements is

$$
C_{n, k}=\frac{n !}{k !(n-k) !}
$$

where $n !=n(n-1)(n-2), \ldots, 1$ and $k !=$ $k(k-1)(k-2), \ldots, 1$. Substituting in (45) $n=5$ and $k=2$ coils, we get $C_{n, k}=10$. This value represents the number of pairs constituted by 14 angular plane sectors positioned in front of the corresponding pairs of coils that we have to consider. Therefore, for each pair of homologous points belonging to such areas, the percentage difference of the magnetic flux density module in such points is calculated. Theoretically, if all these differences are equal to zero, then check 1) above stated is positive, in the sense that the magnitude of the field does not change when we move from one point to a homologous one. In practice, from a numerical point of view, as indicated in 1), we calculate the absolute value of the maximum percentage difference found between the moduli of the magnetic flux densities for all the pairs of homologous points where the same field is evaluated. If this value is sufficiently close to zero, then this first check of symmetry of the field is satisfied. Successively, the ten pairs of angular sectors on which to consider the homologous points were identified. Table IV shows such pairs: each of them is identified by the letters $s$ and $t$ that represent the number of coils in front of which the 14 angular sectors are positioned. These sectors contain the points where the field is calculated (see Fig. 15). With reference to Table IV and Fig. 15, the moduli of the

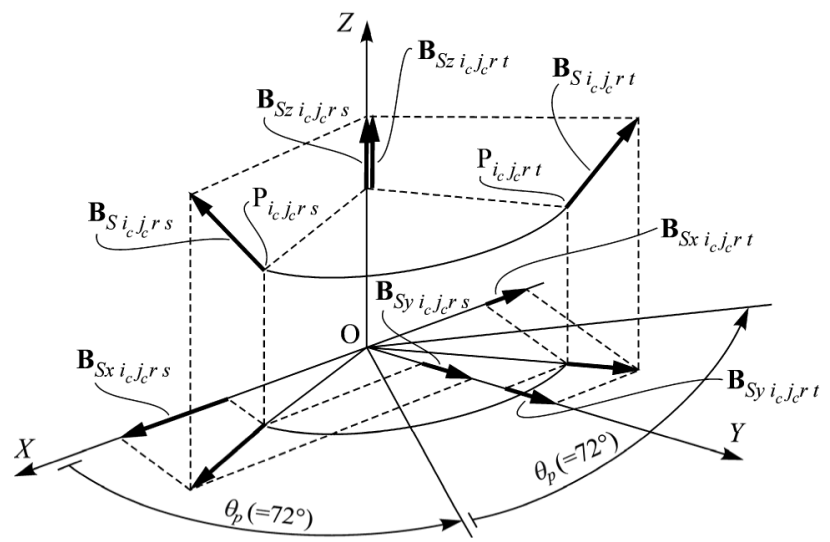

Fig. 16. Magnetic flux densities $\mathbf{B}_{S i_{c} j_{c} r t}$ and $\mathbf{B}_{S i_{c} j_{c} r s}$ in the respective homologous points $P_{i_{c} j_{c} r t}$ and $P_{i_{c} j_{c} r s}$.

magnetic flux densities $\mathbf{B}_{\boldsymbol{S i}_{c} j_{c} r t}$ and $\mathbf{B}_{S i_{c} j_{c} r s}$ were evaluated. Successively, the absolute value of the percentage changes $d_{i_{c} j_{c} r t s}$ of the moduli of the vectors relative to the homologous points $P_{i_{c} j_{c} r t}$ and $P_{i_{c} j_{c} r s}$ were computed

$$
d_{i_{c} j_{c} r t s}=\left|\frac{\left|\mathbf{B}_{S i_{c} j_{c} r t}\right|-\left|\mathbf{B}_{S_{i_{c} j_{c} r s}}\right|}{\left|\mathbf{B}_{S_{i_{c} j_{c} r} r}\right|}\right| \times 100 .
$$

The subscript $r$ identifies the angular plane sectors $r$ th $(r=1,2, \ldots, 14)$ placed in front of the $t$ th and the sth coils. $i_{c}=1,2, \ldots, 5$ and $j_{c}=1,2, \ldots, 4$ identify the homologous points $P_{i_{c} j_{c} r t}$ and $P_{i_{c} j_{c} r s}$ belonging to the $r$ th sectors where $\mathbf{B}_{\boldsymbol{S i}_{c} j_{c} r t}$ and $\mathbf{B}_{\mathbf{S i}_{c} j_{c} r s}$ are calculated, respectively. In (28), the term $n_{\text {sec }}$ exactly represents $r$, i.e., the subscript previously defined. Nevertheless, in order to avoid confusion with the radius $r$ considered in the introduction, in (28), the letter $r$ was not used. Considering the ten pairs of values $(s, t)$ shown in Table IV, with $i_{c}=1,2, \ldots, 5$ and $j_{c}=1,2, \ldots, 4$, by (46), we obtain $2800(=14 \times 20 \times 10)$ values of $d_{i_{c} j_{c} r t s}$. The maximum value found among these 2800 values is equal to $0.00351649 \%$. Therefore, in relation to the moduli of the magnetic flux densities generated by the magnet, the axial symmetry is confirmed. Fig. 16 qualitatively shows the arrangement of two magnetic flux densities vectors relative to two homologous points belonging to the two sectors $r$ th positioned in front of the coils 1 and $2(s=1$ and $t=2)$, respectively.

\section{B. Verification of the Field Components Invariability With Respect to a Relative Reference System}

In order to perform this check, it is necessary to rotate the generic vector $\mathbf{B}_{S i_{c} j_{c} r u}$ by an angle $u \theta_{p}$ around the $Z$-axis $\left(i_{c}=1,2, \ldots, 5, j_{c}=1,2, \ldots, 4, r=1,2, \ldots, 14\right.$, $u=1,2, \ldots, 5)$. Thus, the components $\mathbf{B}_{S x i_{c} j_{c} r u}^{\prime}, \mathbf{B}_{\text {Sy }_{i_{c} j_{c} r u}}^{\prime}$, and $\mathbf{B}_{S z i_{c} j_{c} r u}^{\prime}$ depending on $u$ and relative to the $O^{\prime}\left(X^{\prime}, Y^{\prime}, Z^{\prime}\right)$ reference system integral to the coil considered, have to be evaluated. These components are those detected by an observer integral to the coil. If the field is symmetrical, the components do not vary when the observer moves from one coil to another. Using the rigid body rotation matrix [35], the moduli with sign $B^{\prime}{ }_{S x i_{c} j_{c} r u}$ and $B^{\prime}{ }_{S y i_{c} j_{c} r u}$ of the respective components 


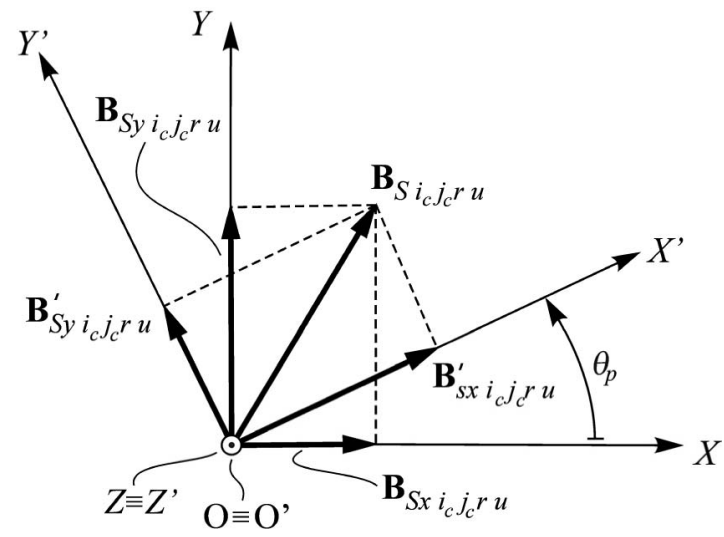

Fig. 17. Computation of the components of the magnetic flux density vector $\mathbf{B}_{S i_{c} j_{c} r u}$ in the reference system $O^{\prime}\left(X^{\prime}, Y^{\prime}, Z^{\prime}\right)$.

TABLE V

Maximum Absolute Percentage Changes OF THE FIELD IN HOMOLOGOUS POINTS

\begin{tabular}{|c|c|c|}
\hline$d_{x i_{c} j_{C}} r t s(\max )$ & $d_{y i_{c} j_{C} r t s(\max )}$ & $d_{z i_{c} j_{C} r t s(\max )}$ \\
\hline $2.24878 \%$ & $0.35078 \%$ & $0.0819493 \%$ \\
\hline
\end{tabular}

$\mathbf{B}_{S x i_{c} j_{c} r u}^{\prime}$ and $\mathbf{B}_{S y i_{c} j_{c} r u}^{\prime}$ relative to the $O(X, Y, Z)$ reference system are obtained (see Fig. 17)

$$
\begin{aligned}
& B_{S x i_{c} j_{c} r u}^{\prime}=B_{S x i_{c} j_{c} r u} \cos \theta_{P}+B_{S y i_{c} j_{c} r u} \sin \theta_{P} \\
& B_{S y i_{c} j_{c} r u}^{\prime}=B_{S y i_{c} j_{c} r u} \cos \theta_{P}-B_{S x i_{c} j_{c} r u} \sin \theta_{P} .
\end{aligned}
$$

Since the axes $Z$ and $Z^{\prime}$ are parallel, independently from $u$ and $\theta_{p}$, we always have

$$
\mathbf{B}_{S z i_{c} j_{c} r u}^{\prime}=\mathbf{B}_{S z i_{c} j_{c} r u} .
$$

Therefore, the corresponding moduli with sign coincide

$$
B^{\prime}{ }_{S z i_{c} j_{c} r u}=B_{S z i_{c} j_{c} r u} \text {. }
$$

Successively, the absolute values of the $8400=2800 \times$ 3 percentage changes of modules with sign relating to the pairs of values $\left(B_{S x i_{c} j_{c} r t}^{\prime}, B_{S x i_{c} j_{c} r S}^{\prime}\right),\left(B_{S y i_{c} j_{c} r t}^{\prime}, B_{S y i_{c} j_{c} r s}^{\prime}\right)$, and $\left(B_{S z i_{c} j_{c} r t}^{\prime}, B_{S z i_{c} j_{c} r s}^{\prime}\right)$ along $X^{\prime}, Y^{\prime}$, and $Z^{\prime}$ in the homologous points $P_{i_{c} j_{c} r t}$ and $P_{i_{c} j_{c} r s}$ were computed in a way similar to that described in Section VII-A

$$
\begin{aligned}
& d_{x i_{c} j_{c} r t s}=\left|\frac{B^{\prime}{ }_{S x i_{c} j_{c} r t}-B^{\prime}{ }_{S x i_{c} j_{c} r s}}{B^{\prime}{ }_{S x i_{c} j_{c} r s} r}\right| \times 100 \\
& d_{y i_{c} j_{c} r t s}=\left|\frac{B^{\prime}{ }_{S y i_{c} j_{c} r t}-B^{\prime}{ }_{S y i_{c} j_{c} r s}}{B^{\prime}{ }_{S y i_{c} j_{c} r s}}\right| \times 100 \\
& d_{z i_{c} j_{c} r t s}=\left|\frac{B^{\prime}{ }_{S z i_{c} j_{c} r t}-B^{\prime}{ }_{S z i_{c} j_{c} r s}}{B^{\prime}{ }_{S z i c_{c} j_{c} r s}}\right| \times 100 .
\end{aligned}
$$

Thus, by (51)-(53), the 8400 values of the percentage changes have been obtained [see the ten pairs of values $(s, t)$ shown in Table IV, with $i_{c}=1,2, \ldots, 5, j_{c}=1,2, \ldots, 4$, and $r=1,2, \ldots, 14]$. In Table $\mathrm{V}$, the maximum value found for each of the three sets of 2800 absolute percentage changes is shown. It is observed that the minimum of these values is associated with the components of the field along the axis $Z^{\prime}\left(d_{z i_{c} j_{c} r t s(\max )}=0.0819493 \%\right)$, while the maximum value is detected in correspondence with the components along the axis $X^{\prime}\left(d_{x i_{c} j_{c} r t s(\max )}=2.24878 \%\right)$. With reference to the components of the field along the axis $Y^{\prime}$, the maximum difference between the components of the field has an intermediate value compared with the two previous values $\left(d_{y i_{c} j_{c} r t s}(\max )=\right.$ $0.35078 \%$ ). In any case, even considering the higher value $d_{x i_{c} j_{c} r t s(\max )}=2.24878 \%$, it is extremely low and the difference with reference to $d_{z i_{c} j_{c} r t s(\max )}=0.0819493 \%$ can certainly be attributed to numerical approximations. These approximations, even if small, affect the calculation of the field components along $X^{\prime}$ and $Y^{\prime}$ in relation to the use of (47) and (48) that contain evaluations of sine and cosine functions (with sums and subtractions of the same ones). These computations, which are not performed when we evaluate the field components along the axis $Z^{\prime}$ [see (50)], certainly introduce numerical errors that, even if very small, affect the final result. With reference to this, one can also qualitatively justify the values $d_{x i_{c} j_{c} r t s(\max )}$ and $d_{y i_{c} j_{c} r t s(\max )}$ significantly higher than the value of $d_{z i_{c} j_{c} r t s(\max )}$ with reference to the particular shape of the magnet considered. As a matter of fact, this magnet has a mass distributed mainly along directions perpendicular to the axis $Z$, i.e., its dimension along $Z$ is small compared with the directions along $X$ and $Y$ (see Fig. 5). Also the plane sectors where we calculate the field have a similar configuration. Consequently, the components of the field along the axes $X$ and $Y$ calculated according to (35), depend on the radial distances (perpendicular to $Z$ ) in a greater measure than the one that affects the components of the same field along $Z$. Therefore, the contributions depending on the numerical evaluations of the distances along $X$ and $Y$ are certainly higher than those associated with the evaluations of the smaller distances along the direction $Z$. Since all the numerical contributions contain errors (truncation, roundoff, and so on), the evaluations of the magnetic flux densities components along $X$ and $Y$ will also be affected by numerical errors greater than those which affect the magnetic flux densities components along $Z$. Moreover, in confirmation of the previous observations, we notice that the integrals which provide the components of the field along $X$ and $Y$, contain the distances along the same $X$-axis and $Y$-axis that are evaluated by products of sine and cosine functions. Conversely, these products do not have to be considered when the distances along $Z$ are computed [see (1)-(3)]. Therefore, the distances along $Z$ are certainly more accurate than those along $X$ and $Y$. This fact definitely causes an increase of the error in the numerical evaluation of integrals that provide the components along $X$ and $Y$. Therefore, the evaluation of the components of the field along $Z$ is more correct and the difference found between the values of $d_{z i_{c} j_{c} r t s(\max )}$ and those of $d_{x i_{c} j_{c} r t s(\max )}$ and $d_{y i_{c} j_{c} r t s(\max )}$ can be justified. Fig. 18 shows the representation of the $1400(=14$ sectors $\times$ 20 points $\times 5$ coils) magnetic flux densities vectors that have been calculated to demonstrate the axial symmetry of the field. The starting point of each vector coincides with the end point of the vector $\mathbf{P}^{\prime}$, whereas the end point of $\mathbf{B}_{S}\left(\mathbf{P}^{\prime}\right)$ has been represented by a dot. Table VI shows the components along $X, Y$, and $Z$ related to the maximum and minimum modulus computed. In order to graphically illustrate the trend of the field, in Fig. 19, the vectors $\mathbf{B}_{S}\left(\mathbf{P}^{\prime}\right)$ concerning the sets of 14 sectors positioned in front of the magnet coil $u=2$ 


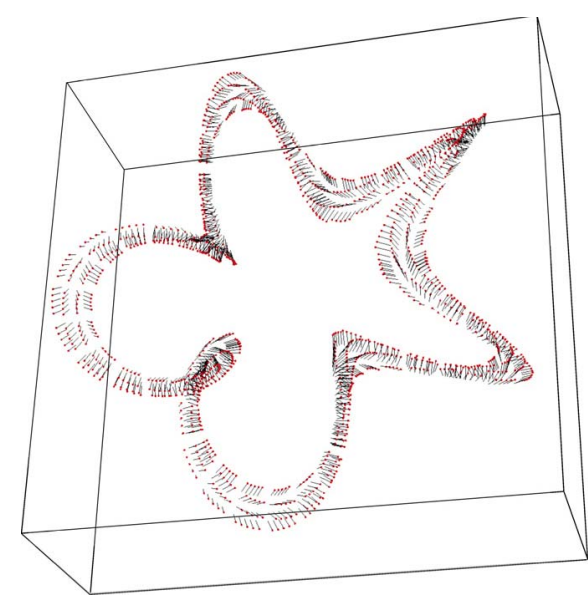

Fig. 18. 1400 magnetic flux densities vectors $\mathbf{B}_{S}\left(\mathbf{P}^{\prime}\right)$.

TABLE VI

Components Relative to the Maximum and Minimum Modulus of the Magnetic Flux Density $\mathbf{B}_{S}\left(\mathbf{P}^{\prime}\right)$

\begin{tabular}{|c|c|c|}
\hline \multicolumn{3}{|c|}{$\begin{array}{l}\max \left|\mathbf{B}_{\boldsymbol{S} i_{c} j_{c}} r u\right|=0.1805 \text { Tesla } \\
\text { with } r=14 \quad u=2 \quad i_{c}=1 \quad j_{c}=3\end{array}$} \\
\hline $\mathrm{B}_{S x i_{c} j_{c} r u}$ & $\mathrm{~B}_{S y i_{c} j_{c} r u}$ & $\mathrm{~B}_{S z i_{c} j_{c} r u}$ \\
\hline \multicolumn{3}{|c|}{ [Tesla] } \\
\hline-0.0785425 & -0.142181 & -0.0787147 \\
\hline \multicolumn{3}{|c|}{$\begin{array}{c}\min \mid \mathbf{B}_{S i_{\mathcal{C}} j_{\mathcal{C}} r u \mid}=0.0829892 \text { Tesla } \\
\text { with } r=5 \quad u=1 \quad i_{c}=1 \quad j_{c}=1\end{array}$} \\
\hline \multicolumn{3}{|c|}{ [Tesla] } \\
\hline $\mathrm{B}_{S x i_{c} j_{c} r u}$ & $\mathrm{~B}_{S y i_{c} j_{c} r u}$ & $\mathrm{~B}_{S z i_{c} j_{c} r u}$ \\
\hline-0.0599284 & 0.0529365 & -0.0222151 \\
\hline
\end{tabular}

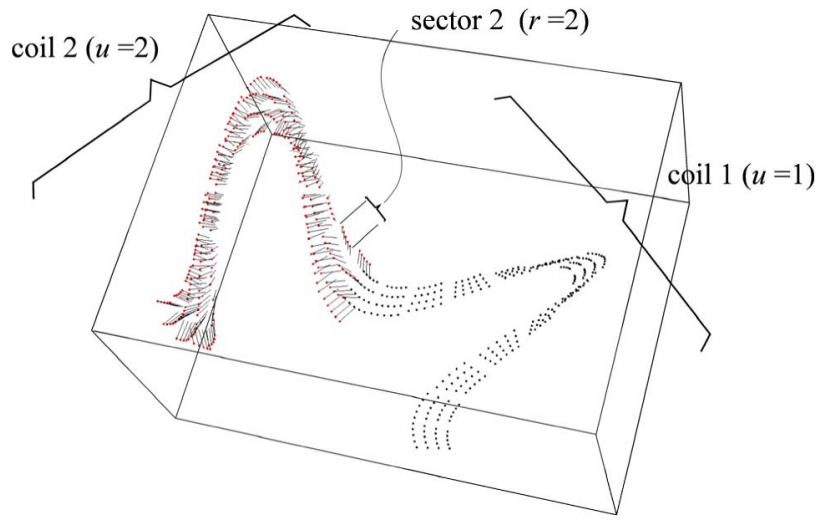

Fig. 19. Vectors $\mathbf{B}_{S}\left(\mathbf{P}^{\prime}\right)$ concerning the sets of 14 sectors positioned in front of the magnet coil $u=2$.

are reported. In this figure, the sector plane $r=2$ is pointed out in detail. In correspondence to the 20 points $P_{i_{c} j_{c} r u}$ with $i_{c}=1,2, \ldots, 5$ and $j_{c}=1,2, \ldots, 4$ belonging to the sector $r=2$, Fig. 20 shows the relative magnetic flux densities vectors $\mathbf{B}_{S i_{c} j_{c} r u}$ (represented in scale). Table VII reports the values of such components.

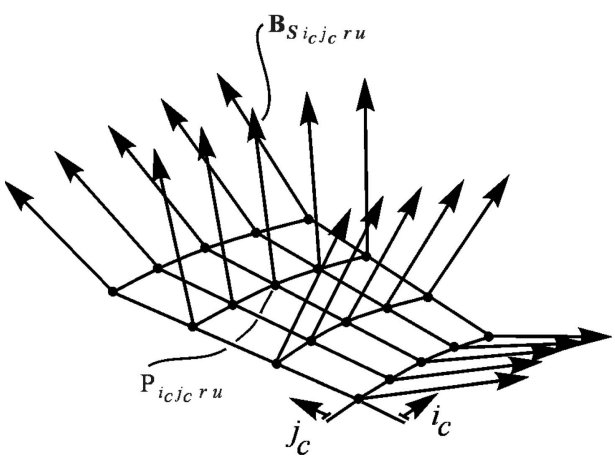

Fig. 20. Magnetic flux density vectors $\mathbf{B}_{\boldsymbol{S i}_{c} j_{c} r u}$ relative to the points of the sector $r=2$ placed in front of the magnet coil $u=2$ (represented in scale).

TABLE VII

Components of the Magnetic Flux Density in the 20 Points of the Angular Plane Sector $u=2$ AND $r=2$

\begin{tabular}{|c|c|c|c|c|}
\hline$i_{c}$ & $j_{c}$ & $\mathrm{~B}_{S x{ }_{c} j_{\mathcal{C}} r u}$ & $\mathrm{~B}_{S y{ }_{C} j_{\mathcal{C}} r u}$ & $\mathrm{~B}_{S z i_{C} j_{\mathcal{C}} r u}$ \\
\hline \multicolumn{2}{|l|}{} & \multicolumn{3}{|c|}{$[$ Tesla] } \\
\hline 1 & 1 & -0.0183887 & 0.0452572 & -0.0262401 \\
\hline 1 & 2 & -0.0582753 & 0.0209651 & -0.0278215 \\
\hline 1 & 3 & -0.0725736 & -0.0184114 & -0.0128356 \\
\hline 1 & 4 & -0.045084 & -0.0633079 & 0.0126235 \\
\hline 2 & 1 & -0.0196055 & 0.0446894 & -0.0307468 \\
\hline 2 & 2 & -0.0592529 & 0.017301 & -0.0286222 \\
\hline 2 & 3 & -0.0702713 & -0.0210366 & -0.00967902 \\
\hline 2 & 4 & -0.0401897 & -0.0574699 & 0.0163837 \\
\hline 3 & 1 & -0.020494 & 0.0438943 & -0.0354014 \\
\hline 3 & 2 & -0.0599789 & 0.0139273 & -0.0294299 \\
\hline 3 & 3 & -0.0680712 & -0.0228386 & -0.00684891 \\
\hline 3 & 4 & -0.0369728 & -0.0521803 & 0.0192174 \\
\hline 4 & 1 & -0.020975 & 0.0430682 & -0.0403678 \\
\hline 4 & 2 & -0.06067 & 0.0108426 & -0.0304355 \\
\hline 4 & 3 & -0.0662898 & -0.024048 & -0.00444419 \\
\hline 4 & 4 & -0.0350004 & -0.047715 & 0.0215503 \\
\hline 5 & 1 & -0.0209584 & 0.0424077 & -0.0459002 \\
\hline 5 & 2 & -0.0615448 & 0.00798433 & -0.0318258 \\
\hline 5 & 3 & -0.0651705 & -0.0249296 & -0.00248418 \\
\hline 5 & 4 & -0.0339758 & -0.0441322 & 0.0237093 \\
\hline
\end{tabular}

\section{NumericAl EVALUATION OF THE FIELD BY AN INTERPOLATION SURFACE}

The magnetostatic field has also been evaluated on each angular plane sectors placed in front of the first coil in a greater number of the previously 20 points/sector considered to study the symmetry of the same field (see Fig. 10). We observe that the second field evaluation was carried out in 625 points/sector. Since there are 14 plane sectors, the field was calculated in $14 \times 625=8750$ points. With reference again to Fig. 10 , in this new evaluation $i_{c}=1,2, \ldots, 25$ and $j_{c}=1,2, \ldots, 25$. The values of $\Delta r$ and $\Delta \varphi$ that define the position of the 625 points have been indicated in Table III (row 2) (see Simulation $n$. 2). By means of this points density (625 points/sector), for each sector, we can consider a suitable interpolating surface that passes through the ends of each of the 625 corresponding magnetic flux densities vectors $\mathbf{B}_{S_{i_{c} j_{c} r u}}(r=1,2, \ldots, 14$ and $u=1$, i.e., we fix the 14 sectors placed in front of the first coil $u=1$ of the magnet). For example, in Fig. 21, $14 \times 625=8750$ 


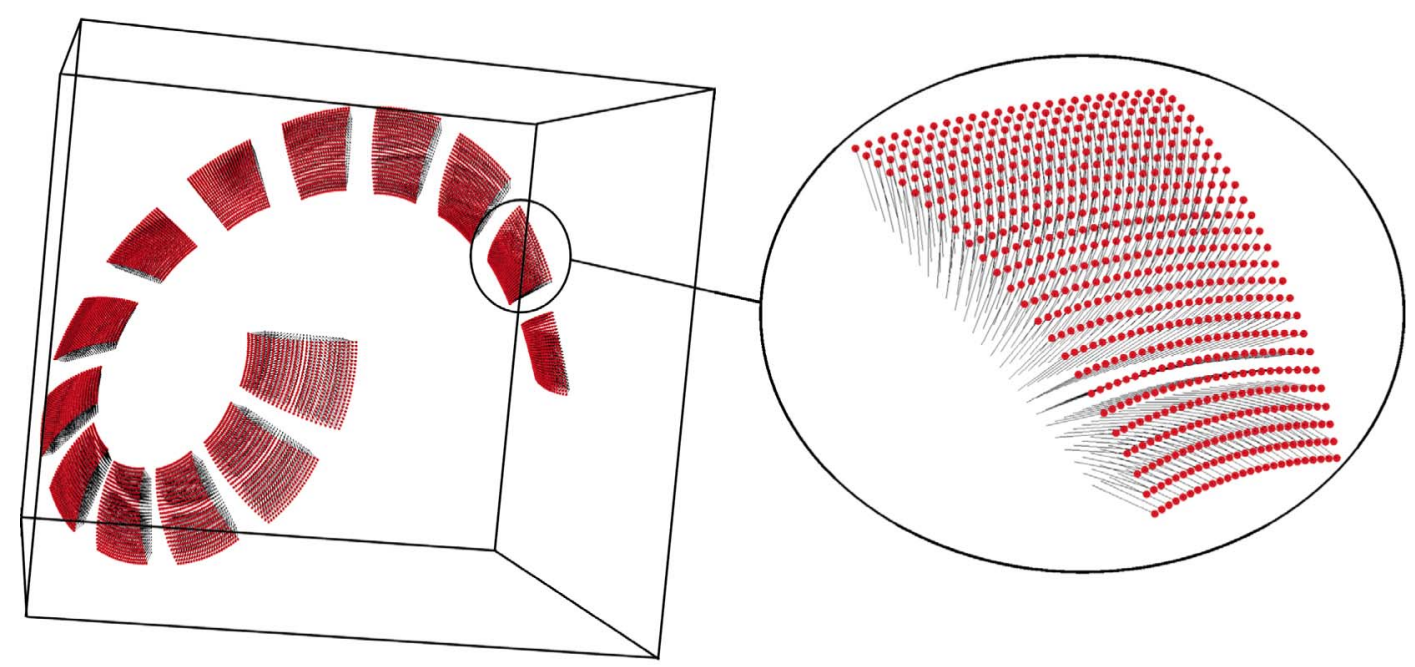

Fig. 21. 8750 magnetic flux density vectors $\mathbf{B}_{S i_{c} j_{c} r u}$ relative to the points belonging to the 14 plane sectors facing the first coil of the magnet $(r=1,2, \ldots, 14$, $u=1, i_{c}=1,2, \ldots, 25$, and $\left.j_{c}=1,2, \ldots, 25\right)$ and magnification of the 625 vettori $\mathbf{B}_{S i_{c} j_{c} r u}$ whose initial points are belonging to the second plane sector $(r=2)$.

TABLE VIII

Comparison of the total Number $n_{t}$ of Points Where $\mathbf{B}_{S}\left(\mathbf{P}^{\prime}\right)$ Is Computed on the Angular Plane Sectors Versus the COMPUTATION TIME $t_{c}$

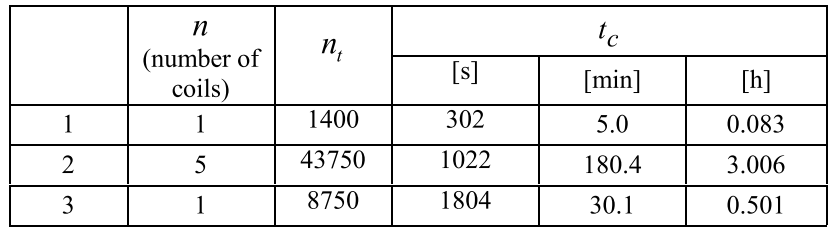

magnetic flux densities vectors are represented. These vectors are relative to the corresponding $14 \times 625=8750$ points previously mentioned. The starting point of such vectors belongs to the 14 plane sectors facing the first coil of the magnet. The end point of the same vectors is represented by a dot. Therefore, the interpolating surface that passes for such dots allows us to interpolate with precision the magnetic flux density in all the infinite points of each sector. If this plane sector represents the surface of the north or south pole of another permanent magnet, it will be possible to perform an accurate and fast calculation, for example, of the magnetic force applied to the surface of the same pole. This force can be calculated by numerically solving the following integral:

$$
\mathbf{F}_{S r u}=\int_{S} \sigma_{M_{r u}}\left(\mathbf{P}^{\prime}\right) \mathbf{B}_{S_{r u}}\left(\mathbf{P}^{\prime}\right) d S
$$

where $\sigma_{M r u}\left(\mathbf{P}^{\prime}\right)$ is the surface charge density in the generic point $P^{\prime}$ of the plane sector $(r, u)$ and $\mathbf{B}_{\boldsymbol{S}_{r u}}\left(\mathbf{P}^{\prime}\right)$ is the magnetic flux density vector in the same point $P^{\prime}$. This magnetic flux density is generated by the toroidal helical magnet and is obtained using the interpolation surface previously mentioned. This kind of interpolation can be obtained very easily, for example, by the software Mathematica [36] or others. Using this technique, it is possible to evaluate the values of the field virtually in all the infinite points of each sector. Therefore, the calculation of the integral indicated in (54) becomes very fast and accurate.

\section{CONCLUSION}

In this paper, the existence of an axial symmetry of the magnetostatic field generated by magnets characterized by toroidal helicoidal geometries is proved. Based on the previous observations and results obtained by the numerical simulations, we notice that this kind of symmetry is valid for all the shapes of helical toroidal magnets similar to those considered in the case study. Since this property depends on the particular orientation of the magnetic flux density vector $\mathbf{B}_{S}\left(\mathbf{P}^{\prime}\right)$ [see (47)-(49) and Fig. 17] and not on the way by which it is generated (magnets and/or corrents), the axial symmetry of a helical toroidal magnetic field is a general characteristic of this kind of field. Consequently, whenever these fields have to be evaluated, whatever the application is (unconventional design configurations of electrical machines, the optimization of magnetic field of synchrotron light sources, tokamaks, stellarator, and so on), we can gain an advantage from a computational point of view. If the magnetization vector $\mathbf{M}$ has a toroidal helical distribution similar to that considered in the case study, regardless of the size and number of coils, the magnetic field generated by the magnet will always show the axial symmetry previously described. In relation to this property, the evaluation of the magnetic flux density in any point around the magnet can be performed by solving the integrals that provide the field in the points of the regions positioned in front of only one coil of the magnet. The immediate practical advantage of this symmetry is a strong reduction of the computation time of the field in the points belonging to all the regions that surround the magnet. As a matter of fact, this field can be simply obtained by rotating the component of the field evaluated in correspondence with the regions located in front of only one magnet coil. In order to illustrate the significant time computation decrease based on the axial symmetry of the field, we can observe Table VIII. This table shows the time computation for different numbers of points where $\mathbf{B}_{S}\left(\mathbf{P}^{\prime}\right)$ is computed. We note that the computation of the field has been performed in 20 points of each angular flat sector (see Figs. 10 and 20). Therefore, the field has been evaluated 
in all the 20 points sets facing the whole helical toroidal magnet surface. The computation time to calculate the field in these 20 points $\times 14$ sectors $\times 5$ coils $=1400$ points by a personal computer Intel I5 is about equal to five minutes [see Table VIII (row 1)]. If we perform the computation in 625 points $\times 14$ sectors $\times 5$ coils $=43750$ points (i.e., in all the angular flat sectors at front of the whole permanent magnet) about $3 \mathrm{~h}$ are necessary [see Table VIII (row 2)]. We can reduce this time computation by considering only a set of 14 flat sectors facing only one magnet coil [in 625 points $\times$ 14 sectors $\times 1$ coil $=8750$ points, computation time: $30 \mathrm{~min}$, see Table VIII (row 3 )]. The axial symmetry of the field allows us to evaluate the field in all the remaining 625 points $\times$ 14 sectors $\times 4$ coils $=35000$ points in a few seconds instead of hours [using (47)-(49), numerical integrations are not necessary]. Therefore, by the axial symmetry of the helical toroidal magnetic field, we can compute it about six times faster, all things being equal. The 14 flat sectors have been considered to define a periodic domain on which to evaluate the field to compute the field exactly in a set of flat positions facing the whole permanent magnet. In relation to possible optimizations, the need to reduce the time computation of the field is important. The field axial symmetry reduces the optimization time and the hardware/software resource can be utilized to increase the computation accuracy.

\section{REFERENCES}

[1] W. F. Brown, Jr., "Electric and magnetic forces: A direct calculation. I," Amer. J. Phys., vol. 19, no. 5, pp. 290-304, 1951.

[2] W. F. Brown, Jr., "Electric and magnetic forces: A direct calculation. II," Amer. J. Phys., vol. 19, no. 6, pp. 333-350, 1951.

[3] R. Muscia, "Equivalent magnetic charge in helicoidal magnets," J. Appl. Phys., vol. 104, no. 10, p. 103916, 2008.

[4] J. P. Selvaggi, S. Salon, O.-M. Kwon, and M. V. K. Chari, "Computation of the three-dimensional magnetic field from solid permanent-magnet bipolar cylinders by employing toroidal harmonics," IEEE Trans. Magn., vol. 43, no. 10, pp. 3833-3839, Oct. 2007.

[5] J. M. M. Rovers, J. W. Jansen, and E. A. Lomonova, "Modeling of relative permeability of permanent magnet material using magnetic surface charges," IEEE Trans. Magn., vol. 49, no. 6, pp. 2913-2919, Jun. 2013.

[6] I. Babuska and W. C. Rheinboldt, "A-posteriori error estimates for the finite element method," Int. J. Numer. Methods Eng., vol. 12, no. 10, pp. 1597-1615, 1978.

[7] J.-F. Remacle, P. Dular, A. Genon, and W. Legros, "A posteriori error estimation and adaptive meshing using error in constitutive relation," IEEE Trans. Magn., vol. 32, no. 3, pp. 1369-1372, May 1996.

[8] O. C. Zienkiewicz and J. Z. Zhu, "The superconvergent patch recovery and a posteriori error estimates. Part 1: The recovery technique," Int. J. Numer. Methods Eng., vol. 33, no. 7, pp. 1331-1364, 1992.

[9] O. C. Zienkiewicz and J. Z. Zhu, "The superconvergent patch recovery and a posteriori error estimates. Part 2: Error estimates and adaptivity," Int. J. Numer. Methods Eng., vol. 33, no. 77, pp. 1365-1382, 1992.

[10] S. Matsutomo, T. Miyamoto, K. Kaneda, S. Noguchi, and H. Yamashita, "An error evaluation scheme based on rotation of magnetic field in adaptive finite element analysis," IEEE Trans. Magn., vol. 42, no. 4, pp. 567-570, Apr. 2006.

[11] E. Creusé, S. Nicaise, Z. Tang, Y. Le Menach, N. Nemitz, and F. Piriou, "Residual-based a posteriori estimators for the A- $\varphi$ magnetodynamic harmonic formulation of the Maxwell system," Math. Models Methods Appl. Sci., vol. 22, no. 4, p. 1150028, 2012.

[12] Z. Tang, Y. Le Menach, E. Creusé, S. Nicaise, F. Piriou, and N. Nemitz, "Residual and equilibrated error estimators for magnetostatic problems solved by finite element method," IEEE Trans. Magn., vol. 49, no. 12, pp. 5715-5723, Dec. 2013.
[13] S. Matsutomo, S. Noguchi, and H. Yamashita, "Adaptive mesh generation method utilizing magnetic flux lines in two-dimensional finite element," IEEE Trans. Magn., vol. 48, no. 2, pp. 527-530, Feb. 2012.

[14] L. Brouwer, S. Caspi, D. Robin, and W. Wan, "3D toroidal field multipoles for curved accelerator magnets," in Proc. PAC, Pasadena, CA, USA, 2013, pp. 907-909.

[15] Y. He et al., "Calculation of the poloidal magnetic field configuration for the J-TEXT tokamak," IEEE Trans. Appl. Supercond., vol. 20, no. 3, pp. 1840-1843, Jun. 2010.

[16] F. Maviglia et al., "Electromagnetic models of plasma breakdown in the JET tokamak," IEEE Trans. Magn., vol. 50, no. 2, Feb. 2014, Art. ID 7023204.

[17] C. G. Jin et al., "Helicon plasma discharge in a toroidal magnetic field of the tokamak," IEEE Trans. Plasma Sci., vol. 39, no. 11, pp. 3103-3107, Nov. 2011.

[18] R. Albanese, G. Artaserse, F. Maviglia, F. Piccolo, and F. Sartori, "Identification of vertical instabilities in the JET tokamak," IEEE Trans. Magn., vol. 44, no. 6, pp. 1650-1653, Jun. 2008.

[19] R. Albanese, M. de Magistris, R. Fresa, F. Maviglia, and S. Minucci, "Numerical formulations for accurate magnetic field flow tracing in fusion tokamaks," in Proc. 9th IET Int. Conf. Comput. Electromagn. (CEM), Mar./Apr. 2014, pp. 1-2.

[20] D. C. Beidler, E. Harmeyer, F. Harmeyer, J. Kisslinger, Y. Igitkhanov, and H. Wobig, "Stellarator fusion reactors-An overview," in Proc. Toki Conf. (ITC), Dec. 2001, pp. 1-6.

[21] P. Probert, "High-performance interpolation of stellarator magnetic fields," IEEE Trans. Plasma Sci., vol. 39, no. 4, pp. 1051-1054, Apr. 2011.

[22] G. H. Neilson et al., "Facilities for quasi-axisymmetric stellarator research," in Proc. IEEE 25th Symp. Fusion Eng. (SOFE), Jun. 2013, pp. 1-6.

[23] S. Imagawa, A. Sagara, and Y. Kozaki, "Conceptual design of magnets with CIC conductors for LHD-type reactors FFHR2m," Plasma Fusion Res., vol. 3, pp. 1-5, 2008, doi: 101585/pfr.3.S1050.

[24] T. Rummel et al., "The superconducting magnet system of the stellarator Wendelstein 7-X," IEEE Trans. Plasma Sci., vol. 40, no. 3, pp. 769-776, Mar. 2012.

[25] A. W. Clark, F. A. Volpe, and D. A. Spong, "Proto-CIRCUS tilted-coil tokamak-stellarator hybrid," in Proc. 25th Symp. Fusion Eng. (SOFE), Jun. 2013, pp. 1-6.

[26] D. Vanoost, H. De Gersem, J. Peuteman, G. Gielen, and D. Pissoort, "Nonlinear magnetostatic finite-element formulation for models with radial symmetry," IEEE Trans. Magn., vol. 50, no. 2, Feb. 2014, Art. ID 7001904.

[27] B. Štumberger, G. Štumberger, D. Dolinar, A. Hamler, and M. Trlep, "Evaluation of saturation and cross-magnetization effects in interior permanent-magnet synchronous motor," IEEE Trans. Ind. Appl., vol. 39, no. 5, pp. 1264-1271, Sep./Oct. 2003.

[28] G. Štumberger, B. Štumberger, and D. Dolinar, "Analysis of crosssaturation effects in a linear synchronous reluctance motor performed by finite elements method and measurements," in Proc. 12th Int. Conf. Power Electron. Motion Control (EPE-PEMC), Aug./Sep. 2006, pp. 1907-1912.

[29] S. Ruoho and A. Arkkio, "Partial demagnetization of permanent magnets in electrical machines caused by an inclined field," IEEE Trans. Magn., vol. 44, no. 7, pp. 1773-1778, Jul. 2008.

[30] R. Fratila, A. Benabou, A. Tounzi, and J. C. Mipo, "Nonlinear modeling of magnetization loss in permanent magnets," IEEE Trans. Magn., vol. 48, no. 11, pp. 2957-2960, Nov. 2012.

[31] R. Muscia, "Computation of the magnetic field generated by helicoidal toroidal permanent magnets," Electromagnetics, vol. 32, no. 1, pp. 8-30, 2012.

[32] D. Vanoost, H. De Gersem, J. Peuteman, G. Gielen, and D. Pissoort, "Two-dimensional magnetostatic finite-element simulation for devices with a radial symmetry," IEEE Trans. Magn., vol. 50, no. 5, May 2014, Art. ID 7400204.

[33] Y. Marechal, B. Ramdane, and D. P. Botelho, "Computational performances of natural element and finite element methods," IEEE Trans. Magn., vol. 50, no. 2, Feb. 2014, Art. ID 7009904.

[34] E. P. Furlani, "A formula for the levitation force between magnetic disks," IEEE Trans. Magn., vol. 29, no. 6, pp. 4165-4169, Nov. 1993.

[35] C. H. Suh and C. W. Radcliffe, Kinematics \& Mechanisms Design. New York, NY, USA: Wiley, 1978.

[36] Mathematica. [Online]. Available:http://www.wolfram.com/mathematica 